\title{
Ising fluids in an external magnetic field: an integral equation approach
}

\author{
I. P. Omelyan, ${ }^{1,2}$ I. M. Mryglod, ${ }^{1,2}$ R. Folk, ${ }^{2}$ and W. Fenz ${ }^{2}$ \\ ${ }^{1}$ Institute for Condensed Matter Physics, 1 Svientsitskii Street, UA-79011 Lviv, Ukraine \\ ${ }^{2}$ Institute for Theoretical Physics, Linz University, A-4040 Linz, Austria
}

(November 20, 2018)

\begin{abstract}
The phase behavior of Ising spin fluids is studied in the presence of an external magnetic field with the integral equation method. The calculations are performed on the basis of a soft mean spherical approximation using an efficient algorithm for solving the coupled set of the Ornstein-Zernike equations, the closure relations, and the external field constraint. The phase diagrams are obtained in the whole thermodynamic space including the magnetic field $H$ for a wide class of Ising fluid models with various ratios $R$ for the strengths of magnetic to nonmagnetic Yukawa-like interactions. The influence of varying the inverse screening lengths $z_{1}$ and $z_{2}$, corresponding to the magnetic and nonmagnetic Yukawa parts of the potential, is investigated too. It is shown that changes in $R$ as well as in $z_{1}$ and $z_{2}$ can lead to different topologies of the phase diagrams. In particular, depending on the value of $R$, the critical temperature of the liquid-gas transition either decreases monotonically, behaves nonmonotonically, or increases monotonically with increasing $H$. The paraferro magnetic transition is also affected by changes in $R$ and the screening lengths. At $H=0$, the Ising fluid maps onto a simple model of a symmetric nonmagnetic binary mixture. For $H \rightarrow \infty$, it reduces to a pure nonmagnetic
\end{abstract}


fluid. The results are compared with available simulations and the predictions of other theoretical methods. It is demonstrated, that the mean spherical approximation appears to be more accurate compared with mean field theory, especially for systems with short ranged attraction potentials (when $z_{1}$ and $z_{2}$ are large). In the Kac limit $z_{1}, z_{2} \rightarrow+0$, both approaches tend to nearly the same results.

PACS number(s): 64.70.Fx, 05.70.Fh, 64.60.-i, 75.50.Mm

\section{INTRODUCTION}

During the past decades, much attention has been paid to the phase behavior of ferromagnetic fluid models with coupled spin and spatial interactions [1-20]. The investigations have been carried out using the mean field (MF) theory [1-7], the method of integral equations [8-13], as well as Monte Carlo (MC) simulation techniques [7,8,11,14-20]. They dealt mainly with simplified models belonging to a so-called ideal class of spin fluids, where the attractive part of nonmagnetic interactions is absent $(R=\infty)$. Moreover, these models were considered, as a rule, in the absence of an external magnetic field $(H=0)$. At finite values of $R$, it has been established $[1,2,4,5]$ that, depending on the relative strength of magnetic interactions, the gas, liquid, paramagnetic, and ferromagnetic states in the system may form phase diagrams of different topologies. For instance, an order-disorder liquid-liquid phase transition may appear additionally to the gas-liquid one.

A complete picture on the phase diagram topology can only be obtained if the field is included $(H \neq 0)$ in the consideration. Then the phase diagram shows the two other critical lines (so called wings) meeting the magnetic transition line in the $H=0$ plane at the tricritical liquid-liquid transition $[4,5]$. The gas-liquid critical point extends to a critical line in the magnetic field. Whether the gas-liquid or liquid-liquid critical line ends in a critical end point and the corresponding other critical line tends to infinite magnetic field depends 
on the model parameters mentioned. This enlarges the number of different "phases" in the global phase diagram (the regions where the different topologies exist in the space of the microscopic model parameters, like interaction strength or range of the potentials).

The global phase diagrams in one-dimensional $R$-domain were obtained for Ising and Heisenberg spin fluids within MF theory [4,5]. To our knowledge, no such diagrams have been investigated up to now using the integral equation method. As is well recognized, the latter method leads to more accurate predictions. It takes into account pair correlations between particles in spin space, which are completely ignored by the MF approach. Previous integral equation studies on magnetic fluids have been restricted exclusively to ideal systems with Heisenberg spin interactions in the absence of an external field $[8,9]$ or only included a few nonzero field values [10-13]. No integral equation calculations have been performed for nonideal spin fluids at $H \neq 0$, even within the well-known Ising model. Note that here we are dealing with genuine fluid models, meaning that the spatial positions of spins are distributed continuously, contrary to simplified lattice gas schemes [21-23], where the spins are positioned on fixed lattice sites.

Due to the discrete character of spin reorientations in the Ising fluid, it can be mapped onto a binary nonmagnetic mixture with symmetric interparticle interactions. In this context, it should be pointed out that in recent years a lot of papers have been devoted to study phase properties in symmetric mixtures by means of MC simulations, the MF theory, as well as the Ornstein-Zernike (OZ) integral equation method [24-31]. Various closure relations, including the standard mean spherical approximation (MSA) [32] and a self-consistent OZ approach (SCOZA) [29,33], have been exploited within the latter method. Note that these studies considered in fact only the case when the chemical potentials of different species are fixed to be equal. In the language of Ising fluids this corresponds to the absence of an external magnetic field.

It has been realized that despite its relative simplicity, the MSA is able to give reliable results for the coexistence phase boundaries including the location of critical points. The accuracy of the MSA scheme gets worse only when calculating critical exponents. On the 
other hand, the more cumbersome SCOZA technique can provide us with highly precise results for the phase boundaries and it remains accurate even near criticality [33]. The other concept is the hierarchical reference theory (HRT) [30,34-36], that combines features of the renormalization group theory (RGT) and theoretical liquid-state approaches and allows to reproduce some critical exponents more precisely with respect to the MSA. For instance, the critical exponent $\beta$ (which gives the curvature of the coexistence curve near the critical point) takes the values $7 / 20$ and 0.345 within the SCOZA and HRT, respectively. They are close to the experimental and RGT prediction $\beta=0.327$, contrary to the MF and MSA value $\beta=1 / 2$ (see section III.B).

However, the SCOZA has so far been implemented only for a restricted class of hardsphere-Yukawa potentials. For these potentials, some solutions within the MSA ansatz can be derived [37-39] in a semianalytical form as a set of nonlinear algebraic equations (which should further be solved numerically). The SCOZA employs such solutions at an intermediate stage of the calculations. In the presence of potentials of any other structure, for instance, of Lennard-Jones (LJ) type or potentials with a soft-core repulsion part, the mathematical structure is less amenable. In addition, the SCOZA enforces the consistency between different thermodynamic routes in a somewhat phenomenological manner by introducing an artificial "temperature" depending on density and concentration. The high level of sophistication of the SCOZA and HRT concepts leads, in turn, to substantial computational problems, when applying them to more realistic interaction potentials. Therefore, for our problem we stick to a variant of the MSA, being well aware of the properties of this approximation near the critical point.

In the present paper, the global phase diagram of the Ising fluid are investigated on the basis of the OZ integral equation method with a soft MSA closure. This allows us to obtain the complete thermal phase diagrams covering the whole range of the relative strength of magnetic interactions and other parameters of the interaction potentials. The dependencies of the critical temperatures and densities on the external field are analyzed in details as well. 


\section{BACKGROUND}

\section{A. The Ising model}

The full potential energy of the Ising fluid can be written in its most general form as

$$
U=\frac{1}{2} \sum_{i \neq j}^{N}\left[\varphi\left(r_{i j}\right)-I\left(r_{i j}\right)-J\left(r_{i j}\right) s_{i} s_{j}\right]-H \sum_{i=1}^{N} s_{i},
$$

where $\mathbf{r}_{i}$ is the (three-dimensional) spatial coordinate of the $i$-th particle carrying spin $s_{i}=$ \pm 1 with $i=1, \ldots, N$ and $N$ being the total number of particles, $r_{i j}=\left|\mathbf{r}_{i}-\mathbf{r}_{j}\right|$ denotes the interspin separation, and $H$ relates to the homogeneous external magnetic field. The exchange integral $(J>0)$ of ferromagnetic interactions and the attraction part $(I>0)$ of nonmagnetic ones can be chosen in the form of Yukawa functions,

$$
\begin{aligned}
& J(r)=\frac{2\left(z_{1} \sigma\right)^{2}}{z_{1} \sigma+1} \frac{\epsilon_{J} \sigma}{r} \exp \left[-z_{1}(r-\sigma)\right], \\
& I(r)=\frac{2\left(z_{2} \sigma\right)^{2}}{z_{2} \sigma+1} \frac{\epsilon_{I} \sigma}{r} \exp \left[-z_{2}(r-\sigma)\right],
\end{aligned}
$$

where $z_{1}$ and $z_{2}$ are the inverse screening lengths of the potentials, $\epsilon_{J}$ and $\epsilon_{I}$ denote the interaction intensities, and $\sigma$ relates to the particle size. The repulsion $\varphi$ between particles can be modeled by a (more realistic) LJ-like soft-core (SC) potential [7],

$$
\varphi(r)=\left\{\begin{array}{cl}
4 \varepsilon\left[\left(\frac{\sigma}{r}\right)^{12}-\left(\frac{\sigma}{r}\right)^{6}\right]+\varepsilon, & r<\sqrt[6]{2} \sigma, \\
0, & r \geq \sqrt[6]{2} \sigma
\end{array}\right.
$$

rather than by the hard-sphere (HS) function

$$
\varphi_{\mathrm{HS}}(r)=\left\{\begin{array}{cc}
\infty, & r<\sigma \\
0, & r \geq \sigma
\end{array}\right.
$$

The multipliers $2\left(z_{1,2} \sigma\right)^{2} /\left(z_{1,2} \sigma+1\right)$, entering in Eq. (2), have been used for the sake of convenience of comparison of our results with previous predictions (see section III). Then, for instance, the integrals $\int_{\sigma}^{\infty} I(r) \mathrm{d} \mathbf{r}=8 \pi \epsilon_{I} \sigma^{3}$ and $\int_{\sigma}^{\infty} J(r) \mathrm{d} \mathbf{r}=8 \pi \epsilon_{J} \sigma^{3}$ are independent of 
$z_{1}$ and $z_{2}$, respectively. Within the standard hard-sphere MF theory (HSMF), such integrals describe the contribution of the interactions to the free energy [3]. Thus, we can say in advance that the HSMF results will not dependent on $z_{1,2}$. At $z_{1,2} \sigma=1$ (the case which is usually considered in theory and simulation), the multipliers go to unity, and we come to the usual form for $I(r)$ and $J(r)$. Within the soft-core MF theory (SCMF), introduced recently in Ref. [7], a slight $z_{1,2}$-dependency should appear. Then the integrals transform to $\int_{0}^{\infty} \exp \left(-\beta \varphi_{\mathrm{SC}}\right)\{I, J\}(r) \mathrm{d} \mathbf{r}=8 \gamma\left(T, z_{1,2}\right) \pi \epsilon_{I, J} \sigma^{3}$, where $\beta^{-1}=k_{\mathrm{B}} T$ denotes the temperature with $k_{\mathrm{B}}$ being Boltzmann's constant, and $\gamma\left(T, z_{1,2}\right)$ is the function which takes into account the softness of repulsion potentials (see Eqs. (8)-(10) of Ref. [7]). For the integral equation approach we expect a more pronounced dependence of the results on $z_{1,2}$.

\section{B. Integral equation approach}

\section{Mapping to a symmetric binary mixture}

Since the spins $s_{i}$ in an Ising fluid take only two values, \pm 1 , we can map the system with $N$ particles carrying spin 1 or -1 onto a binary mixture with $N_{a}$ and $N_{b}$ particles of type $a$ and $b$, respectively, where $N_{a}+N_{b}=N$. Then Eq. (1) transforms to the equivalent form

$$
U=\frac{1}{2} \sum_{i \neq j}^{N_{a}} \phi_{a a}\left(r_{i j}\right)+\frac{1}{2} \sum_{i \neq j}^{N_{b}} \phi_{b b}\left(r_{i j}\right)+\sum_{i, j=1}^{N_{a}, N_{b}} \phi_{a b}\left(r_{i j}\right)-H M
$$

where $M=\sum_{i=1}^{N} s_{i}=N_{a}-N_{b}$ relates to the total magnetization of the system and

$$
\begin{aligned}
& \phi_{a a}(r)=\phi_{b b}(r)=\varphi(r)-[I(r)+J(r)], \\
& \phi_{a b}(r)=\phi_{b a}(r)=\varphi(r)-[I(r)-J(r)]
\end{aligned}
$$

describe the interactions between like and unlike particles in the mixture.

In a further step, we have to rewrite the energy, the magnetic field, and the magnetization per particle $m=M / N$ in terms of appropriate variables suitable for the mixture. Apart from the total number density $\rho=N / V$, where $V$ denotes the volume, such variables include the particle concentration $x$ and the chemical potentials. One has the concentration relations 


$$
x=\frac{N_{a}}{N}, \quad 1-x=\frac{N_{b}}{N}, \quad m=2 x-1 .
$$

In addition, we emphasize that due to a finite value of the external field term on the righthand side of Eq. (5), we have to deal with a mixture prepared in an unusual way. Indeed, when transferring one particle from species $a$ to species $b$ - in the Ising liquid this amounts to flipping a spin from up to down and thus $\Delta M=-2-$ without changing the spatial coordinates, the total change in energy is equal to $\Delta U=2 H$ (the other terms do not contribute to $\Delta U$ due to the symmetry $\phi_{a a}=\phi_{b b}$ of the particle interactions). On the other hand, the change in energy of the mixture is given by

$$
\Delta U=\Delta N_{a} \mu_{a}+\Delta N_{b} \mu_{b}=\mu_{b}(\rho, x, T)-\mu_{a}(\rho, x, T)
$$

where $\mu_{a}$ and $\mu_{b}$ are the chemical potentials of species $a$ and $b$, respectively. This leads to the identification

$$
\Delta \mu \equiv \mu_{b}(\rho, x, T)-\mu_{a}(\rho, x, T)=2 H
$$

Condition (9) can be considered as an additional constraint imposed on the concentration at given values of $\rho, T$, and $H$, namely, $x=x(\rho, T, H)$. The reason for this procedure is the following. In the case of a magnetic fluid, the external field (not the magnetization) is accessible to experiment, whereas for a mixture it is the concentration (note the chemical potentials). In order to study the Ising fluid in the notation of binary mixture one, therefore, has to fix the difference of the chemical potentials.

\section{Formulation of the integral equations}

For mixtures, the OZ integral equations have [32] the form

$$
h_{\alpha \beta}(r)=c_{\alpha \beta}(r)+\sum_{\gamma=a, b} \rho_{\gamma} \int c_{\alpha \gamma}\left(\left|\mathbf{r}-\mathbf{r}^{\prime}\right|\right) h_{\gamma \beta}\left(r^{\prime}\right) \mathrm{d} \mathbf{r}^{\prime},
$$

where the total $h_{\alpha \beta}$ and direct $c_{\alpha \beta}$ correlation functions for a pair of particles of species $\alpha$ and $\beta$ will depend only on their separation distance, $\rho_{\gamma}=N_{\gamma} / V$ is the particle number 
density of the $\gamma$-th species, and the indices $\alpha, \beta$ and $\gamma$ accept two values, $a$ (spin up) and $b$ (spin down).

Eq. (10) must be complemented by an (approximate) closure relation to be solved with respect to $h_{\alpha \beta}$ and $c_{\alpha \beta}$. The standard MSA scheme [32], proposed originally [40] for systems with HS repulsion (4), should be replaced in our case by the soft MSA ansatz (SMSA) $[41,42]$, appropriate for a SC potential (3). It reads

$$
g_{\alpha \beta}(r)=\exp \left[-\beta \phi_{\alpha \beta}(r)+h_{\alpha \beta}(r)-c_{\alpha \beta}(r)+B_{\alpha \beta}(r)\right],
$$

where $g_{\alpha \beta}(r)=h_{\alpha \beta}(r)+1$ denotes the radial distribution function, and

$$
B_{\alpha \beta}(r)=\ln \left[1+s_{\alpha \beta}(r)\right]-s_{\alpha \beta}(r)
$$

is the bridge function with

$$
s_{\alpha \beta}(r)=h_{\alpha \beta}(r)-c_{\alpha \beta}(r)-\beta \phi_{\alpha \beta}^{1}(r)
$$

(no confusion may arise between the index and the Boltzmann factor $\beta$ ). Formally setting $B=0$ in Eq. (11) leads to the hypernetted-chain (HNC) approximation [32].

The SMSA additionally requires the separation of the total potential $\phi_{\alpha \beta}$ in its shortand long-ranged parts $\phi_{\alpha \beta}^{\mathrm{s}}$ and $\phi_{\alpha \beta}^{1}$ with $\phi_{\alpha \beta}=\phi_{\alpha \beta}^{\mathrm{s}}+\phi_{\alpha \beta}^{1}$. There is no general procedure to perform such a separation uniquely for arbitrary potentials. Since the SMSA itself is not exact, the Yukawa potentials in the region of core repulsion $r \lesssim \sigma$ allow splitting to some extent in various ways, leading to various versions of the SMSA. Usually the splitting is carried out by introducing a switch function. One choice among others is to extract the long-ranged part using the Boltzmann-like switching exponent built on the soft-core potential, i.e.,

$$
\phi_{\alpha \beta}^{1}(r)=-[I(r) \pm J(r)] \exp [-\beta \varphi(r)] .
$$

Such an extraction is quite natural, because for $r>\sigma$ the function $\phi_{\alpha \beta}^{1}(r)$ rapidly tends with increasing $r$ to the Yukawa potential $-[I(r) \pm J(r)]$ (we note that $\exp [-\beta \varphi(r)]=1$ for 
$r \geq \sqrt[6]{2} \sigma$, whereas $\left.\lim _{r \rightarrow 0} \exp [-\beta \varphi(r)]=0\right)$. By the replacement $\varphi(r) \rightarrow \varphi_{\mathrm{HS}}(r)$, we come to the standard HS MSA with $h_{\alpha \beta}(r)=0$ for $r<\sigma$ and $c_{\alpha \beta}(r)=\beta[I(r) \pm J(r)]$ for $r \geq \sigma$.

Another trick lies in a modification of the bridge function to the form of Eq. (12) if $s_{\alpha \beta}(r)>0$ and to $B_{\alpha \beta}(r)=0$ when $s_{\alpha \beta}(r) \leq 0$, that combines the SMSA with the HNC approximation. This is in the spirit of the $\mathrm{KH}$ closure proposed by Kovalenko and Hirata $[43,44]$. Note that the pure SMSA sometimes leads to unphysical $r$-domains with negative values of $g(r)$. The modified SMSA preserves by construction the positiveness of $g(r)$ everywhere in density and temperature space.

\section{Calculation of thermodynamic quantities}

Eqs. (10) and (11) constitute a system of six nonlinear integro-algebraic equations for the same number of unknowns $\{h, c\}_{a a},\{h, c\}_{b b}$, and $\{h, c\}_{a b}=\{h, c\}_{b a}$. Once the solutions are found, the thermodynamic quantities are calculated in a straightforward way. In particular, the pressure can be calculated from the virial equation of state

$$
P(\rho, x, T)=\rho k_{\mathrm{B}} T-\frac{2 \pi}{3} \sum_{\alpha, \beta}^{a, b} \rho_{\alpha} \rho_{\beta} \int_{0}^{\infty} \frac{\mathrm{d} \phi_{\alpha \beta}}{\mathrm{d} r} g_{\alpha \beta}(r) r^{3} \mathrm{~d} r,
$$

where $\rho=\rho_{a}+\rho_{b}$. Although the energy and compressibility routes can also be used, we will prefer the virial route (15) because it is most easily implemented.

The chemical potentials can be written in the form

$$
\mu_{\alpha}=\mu_{\alpha}^{*}+k_{\mathrm{B}} T\left(\ln \varrho_{\alpha}+3 \ln \Lambda_{\alpha}\right)
$$

where $\alpha=a, b$ and $\Lambda_{\alpha}$ being the de Broglie thermal wavelength (which is independent of $\rho$ and $x$ ). Explicit expressions for the excess part of $\mu_{\alpha}$ can be derived using the (exact) Kirkwood formula [45]

$$
\mu_{\alpha}^{*}=\sum_{\beta=a, b} \rho_{\beta} \int_{0}^{1} \mathrm{~d} \lambda \int_{0}^{\infty} g_{\alpha \beta}(r, \lambda) \frac{\partial \varphi_{\alpha \beta}(r, \lambda)}{\partial \lambda} 4 \pi r^{2} \mathrm{~d} r .
$$

Here, the integration over $\lambda$ corresponds to the computation of the work of transferring a separate particle from a vacuum $\left(\lambda=0\right.$ with $\left.\varphi_{\alpha \beta}(r, \lambda)=0\right)$ to the system $(\lambda=1$ and 
$\left.\varphi_{\alpha \beta}(r, \lambda)=\varphi_{\alpha \beta}(r)\right)$. Performing the $\lambda$-integration in a manner similar to that proposed in Refs. [43,44], one obtains, taking into account Eqs. (10) and (11) that

$$
\begin{aligned}
\mu_{\alpha}^{*}= & k_{\mathrm{B}} T \sum_{\beta=a, b} \rho_{\beta} \int_{0}^{\infty}\left[\frac{1}{2} h_{\alpha \beta}^{2}(r)-\frac{1}{2} h_{\alpha \beta}(r) c_{\alpha \beta}(r)-c_{\alpha \beta}(r)\right. \\
& \left.+B_{\alpha \beta}(r) g_{\alpha \beta}(r)-\frac{h_{\alpha \beta}(r)}{s_{\alpha \beta}(r)} \int_{0}^{s_{\alpha \beta}(r)} B\left(s^{\prime}\right) \mathrm{d} s^{\prime}\right] 4 \pi r^{2} \mathrm{~d} r
\end{aligned}
$$

with $\int_{0}^{s} B\left(s^{\prime}\right) \mathrm{d} s^{\prime}$ being equal to $(1+s) \ln (1+s)-s(s+2) / 2$ at $s>0$ or 0 for $s \leq 0$.

It is worth mentioning that since the correlation functions are calculated approximately, the above expressions (15) and (18) for the pressure and chemical potentials will not be thermodynamically self-consistent. In particular, the virial, energy and compressibility routes will lead to different results. Although the differences are, as a rule, relatively small, they may distort the phase coexistence properties near a critical point. A derivation of the selfconsistent expressions in the case of the nonideal Ising model at the presence of the external field is a non-trivial problem that needs a separate serious investigation. It could be solved, for instance, within the SCOZA by extending its present implementation from hard-sphere to soft-core repulsion potentials. In this paper for the sake of simplicity we will use the virial pressure complemented by an appropriately chosen Maxwell construction (see subsection II. C).

\section{Phase separations}

For a general binary mixture, the densities $\rho^{\mathrm{I}, \mathrm{II}}$ and concentrations $x^{\mathrm{I}, \mathrm{II}}$ of coexisting phases I and II are determined at a given temperature $T$ from the well-known mechanical and chemical equilibrium conditions

$$
\begin{aligned}
& P\left(\rho^{\mathrm{I}}, x^{\mathrm{I}}, T\right)=P\left(\rho^{\mathrm{II}}, x^{\mathrm{II}}, T\right), \\
& \mu_{a}\left(\rho^{\mathrm{I}}, x^{\mathrm{I}}, T\right)=\mu_{a}\left(\rho^{\mathrm{II}}, x^{\mathrm{II}}, T\right) \equiv \mu_{a}^{\mathrm{I}, \mathrm{II}}, \\
& \mu_{b}\left(\rho^{\mathrm{I}}, x^{\mathrm{I}}, T\right)=\mu_{b}\left(\rho^{\mathrm{II}}, x^{\mathrm{II}}, T\right) \equiv \mu_{b}^{\mathrm{I}, \mathrm{II}} .
\end{aligned}
$$

In our case, they should be complemented by the condition 


$$
\mu_{b}^{\mathrm{I}, \mathrm{II}}-\mu_{a}^{\mathrm{I}, \mathrm{II}}=2 H
$$

following from the external field constraint (Eq. (9)) for each phase. It is convenient to replace $\mu_{a}$ and $\mu_{b}$ by the sum

$$
\mu \equiv \frac{\mu_{a}+\mu_{b}}{2}
$$

and the difference $\Delta \mu$ defined in (9). Then two of the three chemical potential conditions in Eqs. (19) and (20) can be rewritten in the equivalent form

$$
\Delta \mu\left(\rho^{\mathrm{I}}, x^{\mathrm{I}}, T\right)=\Delta \mu\left(\rho^{\mathrm{II}}, x^{\mathrm{II}}, T\right)=2 H .
$$

These conditions will be satisfied automatically, provided the integro-algebraic equations (10) and (11) are solved in conjunction with Eq. (9). Then one finds a consistent set of correlation functions together with the solution

$$
x=x(\rho, T, H)
$$

for the concentration in the mixture. For the Ising fluid, the magnetization $m(\rho, T, H)$ can easily be reproduced from $x$, whenever it is necessary, using relation (7). Solution (23) can now be inserted into the remaining conditions yielding

$$
\begin{aligned}
& P\left(\rho^{\mathrm{I}}, T, H\right)=P\left(\rho^{\mathrm{II}}, T, H\right), \\
& \mu\left(\rho^{\mathrm{I}}, T, H\right)=\mu\left(\rho^{\mathrm{II}}, T, H\right),
\end{aligned}
$$

where the mapping from $(\rho, x, T)$-space to the new set $(\rho, x(\rho, T, H), T) \equiv(\rho, T, H)$ has been performed.

Relations (24) look now like the coexistence conditions for a one-component fluid. Indeed, the Gibbs free energy of the mixture $G=\mu_{a} N_{a}+\mu_{b} N_{b}$ can be rewritten in terms of $N=$ $N_{a}+N_{b}$ and $M=N_{a}-N_{b}$ as $G=\frac{\mu_{a}+\mu_{b}}{2} N-\frac{\mu_{b}-\mu_{a}}{2} M \equiv \mu N-H M$, so that the introduced quantity $\mu$ (Eq. (21)) has the meaning of the chemical potential of the Ising fluid. It can be calculated using expressions (16) and (18) [which were already used when solving 
the external field constraint (see Eqs. (9) and (23)]. Alternatively, a Maxwell-construction scheme has been utilized. In order to demonstrate that this scheme can be applied in its standard form to the magnetic fluid, let us consider the change in the Gibbs free energy. According to the thermodynamic relations, we have $\mathrm{d} G=-S \mathrm{~d} T+V \mathrm{~d} P+\mu_{a} \mathrm{~d} N_{a}+\mu_{b} \mathrm{~d} N_{b} \equiv$ $-S \mathrm{~d} T+V \mathrm{~d} P+\mu \mathrm{d} N-H \mathrm{~d} M$, where $S$ denotes the entropy. During the isothermal $(\mathrm{d} T=0)$ process I $\rightarrow$ II we obtain, integrating by parts, that for the system with a fixed number of particles $(\mathrm{d} N=0)$ in the presence of a constant external field $H$, the free energy increment is equal to $\Delta G=\left.P V\right|_{\mathrm{I}} ^{\mathrm{II}}-\int_{\mathrm{I}}^{\mathrm{II}} P \mathrm{~d} V-H\left(M_{\mathrm{II}}-M_{\mathrm{I}}\right)$. On the other hand, from the definition of $G$ it follows that $\Delta G=\left.N \mu\right|_{\mathrm{I}} ^{\mathrm{II}}-H\left(M_{\mathrm{II}}-M_{\mathrm{I}}\right)$. Since the increment caused by the change $H\left(M_{\mathrm{II}}-M_{\mathrm{I}}\right)$ in the magnetic energy is the same for both routes, we come to the Maxwell construction $Q_{\mathrm{I}, \mathrm{II}}=\left(1 / \rho^{\mathrm{II}}-1 / \rho^{\mathrm{I}}\right) \mathcal{P}+\int_{\rho^{\mathrm{I}}}^{\rho^{\mathrm{II}}} P(\rho, T, H) \mathrm{d} \rho / \rho^{2}=0$, where $\mathcal{P}$ denotes the coexistence pressure. The construction guarantees that the chemical potential will be the same $\left(\left.\mu\right|_{\mathrm{I}} ^{\mathrm{II}}=0\right)$ in both phases I and II, so that the second line of Eq. (24) transforms to $Q\left(\rho^{\mathrm{I}}, \rho^{\mathrm{II}}, T, H\right)=0$.

In such a way, the gas-liquid and liquid-liquid phase transitions of the first order can be determined. The second-order para-ferro magnetic transition at $H=0$ can be found as a boundary (Curie) curve $T_{\lambda}(\rho)$. Below this curve, i.e., for $T<T_{\lambda}(\rho)$, Eq. (9), being solved at $H=0$ with respect to the concentration $x$ (see Eq. (23)), should have a nontrivial solution $x=x(\rho, T, 0) \neq 1 / 2$ (i.e., $m \neq 0)$. For $T>T_{\lambda}(\rho)$, only the trivial one should satisfy Eq. (9) at $H=0$. Note that the trivial solution $x=1 / 2($ or $m=0)$ appears as a result of the symmetry of the interparticle potentials (Eq. (6)). For the same reason, the coexistence phases will have an identical density at concentrations $x$ and $1-x$, or at magnetizations $m$ and $-m$. From the structure of Eqs. (9), (15) and (18) it also follows that, if $x$ (or $m$ ) is a solution to equation (9) at some value of $H$, then $1-x$ (or $-m$ ) will automatically satisfy this equation at the field $-H$. Therefore, the phase diagrams will be symmetric with respect to the magnetic field $H$. 


\section{NUMERICAL CALCULATIONS}

\section{A. Computational algorithm}

The set of OZ integral equations (10) was first reduced to a system of linear algebraic ones, $h_{\alpha \beta}(k)=c_{\alpha \beta}(k)+\sum_{\gamma=a, b} \rho_{\gamma} c_{\alpha \gamma}(k) h_{\gamma \beta}(k)$, by applying the 3-dimensional Fourier transform $A(k)=\int_{V} A(r) \exp (\mathrm{ik} \cdot \mathbf{r}) \mathrm{d} \mathbf{r}=\int_{0}^{\infty} 4 \pi r^{2} A(r) \sin (k r) /(k r) \mathrm{d} r$, where $A$ is any function of $r$. It can be presented in the compact $(2 \times 2)$ matrix form $\mathbf{h}(k)=\mathbf{c}(k)+\mathbf{c}(k) \boldsymbol{\rho} \mathbf{h}(k)$ with $\boldsymbol{\rho}$ being the diagonal density matrix having nonzero elements $[\boldsymbol{\rho}]_{11}=\rho_{a} \equiv x \rho$ and $[\boldsymbol{\rho}]_{22}=\rho_{b} \equiv(1-x) \rho$.

Because of the nonlinearities in the SMSA closure (Eqs. (11)-(14)) and the external field constraint (FC) [see Eqs. (9) and (18)], the coupled set of OZ/SMSA/FC equations have to be solved iteratively. The iterations have been carried out by adapting the method of modified direct inversion in the iterative subspace (MDIIS) [46]. At given values of $\rho, T$, and $H$ the iteration starts from initial guesses for $c_{\alpha \beta}(r)$ and $x$, and the Fourier transformed functions $c_{\alpha \beta}(k)$ are calculated. Then the total correlation functions in $k$-space are obtained analytically, $\mathbf{h}(k)=[\mathbf{I}-\mathbf{c}(k) \boldsymbol{\rho}]^{-1} \mathbf{c}(k)$, where $\mathbf{I}$ denotes the unit matrix. Applying the backward Fourier transform to $\mathbf{h}(k)$ yields $\mathbf{h}(r)=1 /(2 \pi)^{3} \int_{0}^{\infty} 4 \pi k^{2} \mathbf{h}(k) \sin (k r) /(k r) \mathrm{d} k$. With the current values of $\mathbf{c}(r), \mathbf{h}(r)$, and $x$, the residuals to the SMSA closure (11) and field constraint (9) are evaluated. Using them, new values of $\mathbf{c}(r)$ and $x$ are updated according to the MDIIS corrections, and the iteration procedure is repeated until the solutions lead to residuals with a relative root mean square magnitude of $10^{-6}$. The coexistence phase densities were then found by applying the Maxwell construction.

The ratio $R$ of the integrated strengths of magnetic to nonmagnetic interactions can be calculated as

$$
R=\frac{\int_{0}^{\infty} 4 \pi g(r) J(r) r^{2} \mathrm{~d} r}{\int_{0}^{\infty} 4 \pi g(r) I(r) r^{2} \mathrm{~d} r}
$$

where $g(r)=x^{2} g_{a a}(r)+2 x(1-x) g_{a b}(r)+(1-x)^{2} g_{b b}(r)$ is the (total) radial distribution

function of the Ising fluid. From the form of Yukawa potentials $I(r)$ and $J(r)$ (Eq. (2)) it follows that the relation (25) transforms to 


$$
R=\frac{\epsilon_{J}}{\epsilon_{I}}
$$

for $z_{1}=z_{2}$, We have preferred, the latter definition even when $z_{1} \neq z_{2}$, because the former is sensitive to the approximation made for $g(r)$.

The strength $\varepsilon$ appearing in the SC potential (Eq. (3)) was set to $\epsilon_{J}$. This corresponds to a moderate softness of $\varphi$ with respect to the total potential (see Eq. (1)). In the presentation of our results we use the dimensionless density $\rho^{*}=\rho \sigma^{3}$, temperature $T^{*}=k_{\mathrm{B}} T / \epsilon_{J}$, external field $H^{*}=H / \epsilon_{J}$, and inverse screening lengths $z_{1,2}^{*}=z_{1,2} \sigma$.

\section{B. Results for the ideal system $(R=\infty)$}

Examples of the phase diagrams obtained within the OZ/SMSA/FC integral equation approach for the soft-core ideal Ising fluid with $z_{1}^{*}=1$ at various values, $H^{*}=0,0.1,0.5$, 1, 5, and $\infty$, of the external field are shown in sets (a), (b), (c), (d), (e), and (f) of Fig. 1, respectively, in the $\left(T^{*}, \rho^{*}\right)$ plane. For the purpose of comparison, the results of the SCMF theory and available MC simulation data [7] are also included in this figure.

As can be seen clearly, the OZ/SMSA/FC approach leads to much more accurate predictions of the liquid-gas coexistence densities with respect to the usual version of the SCMF theory. Even the adjustable version, when a semiphenomenological parameter is introduced within the SCMF and fitted to MC data at $H \rightarrow \infty$ (see Ref. [7]), provides us with worse results. At the same time, the deviations between the OZ/SMSA/FC predictions and MC data are relatively small, especially for regions which are well below the critical point. On the other hand, the precision decreases when approaching the criticality point, where the uncertainties in critical temperature and density estimations can reach about 10-15\%. Moreover, the computations have shown that the OZ/SMSA/FC approach reproduces, like the MF theory, the classical values of critical exponents. For instance, the density difference in

liquid and gas phases, $\rho_{\mathrm{L}}^{*}-\rho_{\mathrm{G}}^{*}$, appears to be proportional near a critical point to $\left(T_{\mathrm{c}}^{*}-T^{*}\right)^{\beta}$ independently of $H^{*}$ with the critical exponent $\beta=1 / 2$ (instead of the values $\beta \approx 1 / 3$ and 7/20 obtained within the RGT and SCOZA, respectively). 
The influence of varying the screening length $z_{1}$ of magnetic interactions on the $\mathrm{OZ} / \mathrm{SMSA} / \mathrm{FC}$ phase diagram is illustrated in Fig. 2. In this respect, it should be emphasized that within the SCMF scheme, the results will depend on $z_{1}$ very weakly (see comments at the end of subsection II.A). Using the more precise integral equation approach, we can observe an obvious $z$-dependence of the binodal for all values of the external field. In particular, the dimensionless critical temperature $T_{\mathrm{c}}^{*}$ and density $\rho_{\mathrm{c}}^{*}$ increase considerably with rising $z_{1}$ at each fixed value of $H$. However, the topology of the phase diagram remains the same and is not affected by varying the screening length. Namely, as in the case of the SCMF theory [7], the OZ/SMSA/FC approach predicts a tricritical point for the ideal Ising fluid at $H^{*}=0$ and not a critical end point besides a gas-liquid critical point. This holds for $z_{1}^{*} \leq 5$ and at least to within the relative accuracy $10^{-6}$ of the numerical calculations.

The change in $z_{1}^{*}$ (this quantity will be denoted below simply as $z^{*}$ ) does not also affect the tendency of the critical temperature (density) to decrease (increase) monotonically with increasing the external field strength $H^{*}$. The dependencies $T_{\mathrm{c}}^{*}\left(H^{*}\right)$ and $\rho_{\mathrm{c}}^{*}\left(H^{*}\right)$ are plotted in detail in subsets (a) and (b) of Fig. 3, respectively. The OZ/SMSA/FC calculations show that in the limit of weak fields, the functions $T_{\mathrm{c}}^{*}\left(H^{*}\right)$ and $\rho_{\mathrm{c}}^{*}\left(H^{*}\right)$ can be cast in the forms $T_{\mathrm{c}}^{*}\left(H^{*}\right)=T_{\mathrm{c}}^{*}(0)-c_{T}\left(z^{*}\right)\left(H^{*}\right)^{2 / 5}$ and $\rho_{\mathrm{c}}^{*}\left(H^{*}\right)=\rho_{\mathrm{c}}^{*}(0)+c_{\rho}\left(z^{*}\right)\left(H^{*}\right)^{2 / 5}$ with $c_{T}\left(z^{*}\right)$ and $c_{\rho}\left(z^{*}\right)$ being quantities depending only on $z^{*}$. The exponent of this power law behavior is in accordance with the mean field tricritical exponent $[4,7]$ (where, of course, $c_{T}$ and $c_{\rho}$ are independent of $\left.z^{*}\right)$. With increasing $H^{*}$, the functions $T_{\mathrm{c}}^{*}\left(H^{*}\right)$ and $\rho_{\mathrm{c}}^{*}\left(H^{*}\right)$ begin to tend rapidly (especially at small and moderate values of $z$ ) to their infinite field limits. For larger $z^{*}$, the saturation regime shifts to higher values of $H^{*}$. At small inverse screening length $\left(z^{*}<0.5\right)$, the OZ/SMSA/FC and SCMF results are practically indistinguishable.

It is worth mentioning that in the Kac limit $z^{*} \rightarrow 0$, the SCMF theory should lead to exact results provided the equation of state of the reference system is chosen exactly too. Indeed, at $z^{*} \rightarrow 0$ the magnitude $2(z \sigma)^{2} /(z \sigma+1)$ of magnetic potential vanishes, whereas the screening length $1 / z$ tends to infinity. Under such conditions, the magnetic interactions can be treated as an infinitesimally small perturbation to the reference potential and the 
assumptions of the MF theory become exact. With increasing $z^{*}$, the precision of the SCMF description goes down. Note that within the standard HSMF and SCMF theories, the reference system relates exclusively to nonmagnetic HS or SC repulsion. This is acceptable for long ranged $\left(z^{*} \lesssim 1\right)$ potentials. When the screening radius is short enough $\left(z^{*} \gtrsim 2\right)$, a more appropriate choice of the reference system (with including a part of the Yukawa potential) should be performed. On the other hand, the accuracy of the OZ/SMSA/FC approach when evaluating $T_{\mathrm{c}}^{*}$ and $\rho_{\mathrm{c}}^{*}$ is expected to be of order of $10-15 \%$, in a wide region of $z^{*}$-values. This is the same accuracy as for the case $z^{*}=1$, where the direct comparison with the MC data could be performed (see Fig. 1).

The OZ/SMSA/FC results for the magnetic phase transition (which takes place only at $\left.H^{*}=0\right)$ are shown in Fig. 4 (a) for the set $z^{*}=0.5,1,2,3$, and 5 of the inverse screening length over a wide temperature and density region. It can be seen that the dependence of the Curie temperature $T_{\lambda}^{*}$ on $\rho^{*}$ shifts considerably to smaller values of $\rho^{*}$ with increasing $z^{*}$. This dependence is nonlinear contrary to the HSMF prediction, where the function $T_{\lambda}^{*}=8 \pi \rho^{*}$ behaves linearly on $\rho$ and independently of $z$. Within the more accurate SCMF and OZ/SMSA/FC approaches, the linear dependence of $T_{\lambda}^{*}$ on $\rho^{*}$ is recovered only in the particular (Kac) limit $z^{*} \rightarrow 0$. At larger values of $z^{*}$ (namely, at $z^{*}>2$ ), the deviations from the limiting behavior become significant. A similar, but considerably weaker $z^{*}$-dependence of $T_{\lambda}^{*}$ to that presented in Fig. 4 (a) for the OZ/SMSA/FC approach is observed within the SCMF theory, due to the existence of factor $\gamma\left(T^{*}, z^{*}\right)$ in the temperature $T_{\lambda}^{*}=8 \pi \gamma \rho^{*}$ (see Ref. [7]). Note also that the difference between the SCMF and OZ/SMSA/FC functions $T_{\lambda}^{*}\left(\rho^{*}\right)$ increases with rising $z^{*}$. However, even for small values of $z^{*}$, where the $z^{*}$-dependence is not so pronounced, the SCMF theory leads to worse predictions. This is demonstrated in Fig. 4 (b) for a particular case $z^{*}=1$ by comparison with MC results. Although here the both theories agree quite well, the SCMF deviations from the MC data are, nevertheless, slightly larger than those of the OZ/SMSA/FC theory. 


\section{Results for nonideal models $(0<R<\infty)$}

\section{Zero magnetic field}

The phase coexistences of the nonideal Ising fluid with $z_{1}^{*}=z_{2}^{*}=1$ are shown in Fig. 5 for $H^{*}=0$, when the ratio $R$ of strengths of the magnetic to nonmagnetic interaction is not too small. As can be seen, all the curves exhibit a tricritical point behavior - type I of the thermodynamic phase diagrams - of the same topology as the case $R=\infty$. Note that because of the great number of curves, the magnetic phase transition lines have been omitted in Fig. 5 as well as in Figs. 6-9. They are presented in detail in Fig. 14 below.

With further decreasing $R$, the shape of the phase diagrams changes in a characteristic way. This is illustrated in subsets (a) and (b) of Fig. 6. Beginning from the upper boundary value $R=R_{u}=0.215$, beside the tricritical point (TCP) a gas-liquid critical point (GLCP) appears in the paramagnetic phase region (at smaller densities than the tricritical point density) indicating that the nonmagnetic interaction is strong enough to condense here the system into the liquid phase. In addition, a triple point becomes visible. The TCP now corresponds to a liquid-liquid transition between paramagnetic and ferromagnetic phases. This is different from the phase behavior of type $\mathrm{I}$ in the region $R_{u}<R \leq \infty$, where the TCP relates to the transition between a paramagnetic gas and a ferromagnetic liquid phase.

Such a new topology of the phase behavior below $R_{u}$ will be referred to as type II. The appearance of the additional critical point with decreasing $R$ is explained by an increased weight of nonmagnetic attractions in the system. The nonmagnetic interaction is sufficiently strong to produce a gas-liquid transition before the liquid becomes ferromagnetic.

If the nonmagnetic interaction becomes too strong, namely, when the value of $R$ is below the lower boundary level $R_{l}$, i.e., $R<R_{l}=0.14$, the TCP disappears and transforms into a critical end point (CEP) (see subset (b) of Fig. 6). At the same time, the GLCP remains and further shifts away from the CEP. Such a topology of the phase diagram in the region $R<R_{l}$ will be defined as of type III. Note that for extremely small values of $R$ (when 
$R \ll R_{l}$ ), the phase coexistence will behave like that inherent in a simple nonmagnetic fluid (because then $I \gg J$ and magnetic interactions can be ignored completely).

These three phase diagram topologies at $H=0$ have been found earlier for other systems, such as symmetric binary nonmagnetic mixtures $[24,25,28,29]$, the Heisenberg fluid $[4,17]$ or the Stockmayer fluid [47]. It is interesting to remark that the boundary values $R_{u}=0.215$ and $R_{l}=0.14$ calculated by us within the OZ/SMSA/FC approach for the Ising fluid correspond to $\delta_{u}=0.646$ and $\delta_{l}=0.754$. Here $\delta=(1-R) /(1+R)$ denotes the ratio of interparticle potentials (outside the hard or soft core) between unlike and like (see Eq. (6), $\delta$ is proportional to the ratio $[I(r)-J(r)] /[I(r)+J(r)])$ particles in the mixture. The latter values are very close to those $\left(\delta_{u}=0.65\right.$ and $\left.\delta_{l}=0.75\right)$ reported in Ref. [29] for a symmetric binary mixture and evaluated within the SCOZA technique (the HRT yields $\delta_{u}=0.665$ [30]). However, the direct comparison is not possible since we used the soft core potential instead of the hard sphere repulsion and another value of the inverse screening length $z \equiv z_{1}=z_{2}$.

Varying the parameter $z^{*}$ can lead to a qualitative modification of the phase diagrams and thus to a shift of the boundaries between different topologies. This is seen in Fig. 7, where the cases $z_{1}^{*}=z_{2}^{*}=0.5,1,2$, and 3 are considered at $R=0.215$ (subset (a)) and $R=0.14$ (subset (b)). From the topologies of these diagrams it can be concluded that the upper $R_{u}\left(z^{*}\right)$ and lower $R_{l}\left(z^{*}\right)$ boundary values decrease with increasing $z^{*} \equiv z_{1}^{*}=z_{2}^{*}$. A more complicated situation arises when $z_{1}^{*} \neq z_{2}^{*}$ that is presented in Fig. 8. Here, the quantities $R_{u}$ and $R_{l}$ should be treated as depending on both inverse screening lengths $z_{1}^{*}$ and $z_{2}^{*}$. Analyzing the set of curves in Fig. 8, it can be stated that the behavior of $R_{u}\left(z_{1}^{*}, z_{2}^{*}\right)$ and $R_{l}\left(z_{1}^{*}, z_{2}^{*}\right)$ is not monotonic. In particular, the functions $R_{u}\left(z_{1}^{*}, z_{2}^{*}\right)$ and $R_{l}\left(z_{1}^{*}, z_{2}^{*}\right)$ increase with rising $z_{2}^{*}$ at fixed $z_{1}^{*}=1$, but they decrease with increasing $z_{1}^{*}$ at constant $z_{2}^{*}=1$.

It is worth mentioning that the above three types of the phase diagrams can also be observed within MF theory [4]. The disadvantage of the MF description is that it produces boundary values $R_{u}$ and $R_{l}$ which are independent of $z_{1}^{*}$ and $z_{2}^{*}$. This corresponds, in fact, to the limiting behavior of $R_{u}$ and $R_{l}$ at $z_{1}^{*}, z_{2}^{*} \rightarrow 0$. 


\section{Nonzero magnetic field}

A set of phase diagrams for different values of the external field $H$ and relative strength $R$ of internal magnetic to nonmagnetic interactions are plotted in Fig. 9. Here, we can see that the change in $H$ modifies considerably the phase coexistence curves in the nonideal Ising system. At large values of $R$, such modifications are similar to those of the ideal Ising fluid (compare, for example, the subset (a) of Fig. 9, $R=5$, with the subset (b) of Fig. 2, $R=\infty)$. Since there is no magnetic phase transition at finite fields, the tricritical point at $H^{*}=0$ transforms into a critical point for $H^{*} \neq 0$ (note that one has a phase diagram symmetric in $H \rightarrow-H)$ and moves monotonically in the temperature-density plane with increasing $H$ to the side of lower $T^{*}$ and higher $\rho^{*}$. This demonstrates that the critical lines meet in a tricritical point. At intermediate and small values of $R$, the phase diagram modifications exhibit nonmonotonic features. For instance, at $R=0.29$ (see subset (c)), the critical temperature, starting from the value $T_{\mathrm{c}}(0)$ at $H=0$, begins first to go down, reaching a minimum at $H^{*} \sim 2$. Further it increases up to its limiting value $T_{\mathrm{c} \infty}=\lim _{H \rightarrow \infty} T_{\mathrm{c}}(H)$, where $T_{\mathrm{c} \infty}$ can be less (subset (c)) or greater (subset $\left.(\mathrm{d})\right)$ than $T_{\mathrm{c}}(0)$.

More complicated scenarios are observed for parameters $R_{l}=0.14<R<0.215=R_{u}$ (the region of topologies of type II), when the gas-liquid critical point exists simultaneously (from the left in $\rho^{*}$-axis) to the tricritical one. However, only one of these two critical points at $H=0$ can be connected by a critical line with the critical point at $H=\infty$. With increasing $H$, one of the two critical lines has to end. This is only possible in a critical end point. How this happens depends on the concrete value of $R$ (see subsets (e), (f), and (g) of Fig. 9). In the infinite field limit $H \rightarrow \infty$, the phase diagrams tend at each $R$ to the gas-liquid binodals of a simple nonmagnetic fluid with the interparticle potential $\phi(r)=\varphi(r)-I(r)-J(r)$. This is because then all the spins align exactly along the field vector, so that the product $\mathbf{s}_{i} \cdot \mathbf{s}_{j}$ will be equal to 1 (see Eq. (1)) for any pair of particles. A similar behavior even for finite fields can be observed for regions with too low values of $R<R_{l}=0.14$ (see subset $(\mathrm{h})$ ), where the influence of magnetic interactions can be neglected 
$(J \ll I)$.

The dependencies of the gas-liquid critical temperature $T_{\mathrm{c}}^{*(g l)}$, the liquid-liquid critical (wing) temperature $T_{\mathrm{c}}^{*(w)}$ and the corresponding densities $\rho_{\mathrm{c}}^{*(g l)}$ and $\rho_{\mathrm{c}}^{*(w)}$ of the nonideal Ising fluid on the value $H^{*}$ of the external field are shown in detail in Figs. 10 and 11, respectively. They cover the whole region of varying $R$ and include all the three types, I (subset (a)), II (subsets (b) and (c)), and III (subset (d)) of the phase diagram topology. Note that the both phase transitions exist only in region II of the global phase diagram, whereas the wing line disappears for $R<R_{l}$ and the gas-liquid phase transition line does not appear for $R>R_{u}$. For type I (subset (a)), the wing line will correspond to the gas-liquid critical point (no liquid-liquid phase transitions are present in $R$-regions corresponding to types I and III). As can be seen, the monotonic decrease of $T_{\mathrm{c}}^{*(w)}$ with rising $H^{*}$, observed at $0.5 \leq R \leq \infty$, gradually transforms into a nonmonotonic function $T_{\mathrm{c}}^{*(w)}\left(H^{*}\right)$, when the parameter $R$ lies in the interval $\left[R_{v l}, 0.4\right]$ with $R_{v l}=0.196$ (subset (a) of Fig. 10). The position of the minimum in $T_{\mathrm{c}}^{*(w)}\left(H^{*}\right)$ shifts from $H^{*} \sim 3$ to 1 with decreasing $R$. At $R \geq R_{v l}$, the wing line can exist for arbitrary fields $0 \leq H^{*} \leq \infty$. For $R<R_{v l}$, the wing line terminates at some finite value $H_{c e}^{*}(R)$ (subset (b) of Fig. 10) until it disappears at $R<R_{l}=0.14$

The wing line density $\rho_{\mathrm{c}}^{*(w)}\left(H^{*}\right)$ also exhibits a nonmonotonic field behavior in the interval $0.6 \leq R<1.25$ with a maximum at $H^{*} \sim 1$ to 2 . Outside of this interval, it increases $(R \geq 1.25)$ or decreases $(R<0.6)$ monotonically (see subsets (a) and (b) of Fig. 11). On the other hand, for $0.196=R_{v l}<R<R_{u}=0.215$ the gas-liquid critical point ends in a critical end point at some finite value $H_{\text {glce }}^{*}(R)$ (subset (b) of Figs. 10 and 11). For $R \leq R_{v l}$, the gas-liquid transition line exists for arbitrary fields $0 \leq H^{*} \leq \infty$. The critical temperature $T_{\mathrm{c}}^{*(g l)}\left(H^{*}\right)$ of this transition increases always monotonically with increasing $H^{*}$ (subsets (c) and (d) of Fig. 10), whereas the critical density $\rho_{\mathrm{c}}^{*(g l)}\left(H^{*}\right)$ is always a nonmonotonic function exhibiting a maximum at $H^{*} \sim 3$ to $H^{*} \sim 30$ depending on $R$ (see subsets (c) and (d) of Fig. 11). 


\section{Van Laar point in the global phase diagram}

From the phase diagram analysis presented it follows that in the region of topologies of type II $\left(R_{l}<R<R_{u}\right)$, the gas-liquid and liquid-liquid transitions can coexist simultaneously not only at $H=0$ but also for $H \neq 0$. On the other hand, it has been realized that for sufficiently strong fields including the limit $H \rightarrow \infty$, we have a simple nonmagnetic-like phase behavior with the presence of only one gas-liquid transition (see Fig. 9). Thus, region II in the global phase diagram has to split into two subregions in dependence whether the gas-liquid (type IIa, $R_{v l}<R<R_{u}$ ) or the liquid-liquid (type IIb, $R_{l}<R<R_{v l}$ ) critical line terminates in a critical end point at some finite value of $H^{*}$. The boundary in the global phase diagram between these two regions defines a van Laar-like point [48].

Our calculations have shown that this special point is identified at $R_{v l}=0.196$ with $H_{t r}^{*} \approx 2.2$ for $\rho_{t r}^{*} \approx 0.55$ and $T_{t r}^{*} \approx 21.3$. As can be seen in Fig. 12 , there exists a some finite

critical value $H_{t r}$, where both the gas-liquid and liquid-liquid critical lines merge in one point in the temperature-density plane projection. It is an asymmetric tricritical point and the change of the critical exponent $\beta$ from the mean field value $(\beta=1 / 2)$ to its tricritical value $(\beta=1 / 4)$ is seen in Fig. 12 (subset $(b))$. The van Laar point has been found in symmetric mixtures [49] but so far not in Ising liquids. In van der Waals theory the value of the van Laar point is $R_{v l}^{\mathrm{MF}}=0.279$ in agreement with the value $\delta=0.564$ given in [49] (note that $\Lambda=1-\delta=0.436$ there). The value $R_{v l}=0.196$ is comparable to that found (at $z^{*}=1.8$ ) in [30] (their $\delta_{v l}=0.67$ corresponds to $R_{v l}=0.197$ ). In order to collect our results for the thermodynamic phase diagrams, the global phase diagram in $R$-space is presented in Fig. 13.

\section{Magnetic critical line}

Let us consider, finally, the OZ/SMSA/FC result on the para-ferro coexistence in the nonideal Ising fluid at $H=0$. It is shown in Fig. 14 for various values of $R$ in a wide region of the temperature-density plane. In the case of the ideal fluid $(R=\infty)$, a strong dependence 
of the Curie temperature $T_{\lambda}^{*}$ on the screening length of the magnetic interaction (see subset (a) of Fig. 4) has been found. As can be observed in Fig. 14, the function $T_{\lambda}(\rho)$ exhibits also an $R$-dependence, especially at low densities. This is in a contrast to predictions of the HSMF and SCMF theories which lead to values of $T_{\lambda}^{*}\left(\rho^{*}\right)$ independent of $R$ (see dashed curves in Fig. 14). However at larger densities $\left(\rho^{*}>1\right)$ all the OZ/SMSA/FC functions begin to converge to the same curve, which is almost a straight line, and the $R$-dependence vanishes. This line does not coincide with the HSMF and SCMF results.

\section{CONCLUSIONS}

We have formulated a generalization of the integral equation formalism for symmetric binary mixtures in order to study phase coexistence properties of Ising spin fluids in the presence of an external magnetic field $H$. Mapping the spin system onto the binary mixture shows that the calculations for the soft-core Ising fluid at a certain magnetic field reduce in the mixture picture to the calculations at a certain value of the chemical potential difference of the constituents of the mixture. This introduces a field constraint to the OZ equations and modifies the MSA closure to the SMSA ansatz. It has been demonstrated that the resulting OZ/SMSA/FC approach is able to describe adequately the phase behavior of such models. Depending on the ratio $R$ of the strengths of the magnetic and nonmagnetic interactions inherent in the spin fluid system, four types of thermodynamic phase diagrams have been identified.

As has been established, the OZ/SMSA/FC approach provides us with more accurate predictions in comparison to those of the MF theory and corroborates the MF global phase diagram, changing of course the boundary values of $R$ which separates the different topolo-

gies. It is expected that some other more complicated schemes, such as the SCOZA, for example, should lead to a higher precision of the calculations. However, they are not yet developed in their present formulations to be directly applied to magnetic fluids with softcore repulsion potentials. Due to their high level of sophistication, they meet considerable 
computational difficulties in actual implementations. On the other hand, the approach proposed here can be used for systems with arbitrary potentials at relatively low computational costs.

The OZ/SMSA/FC scheme can also be extended to magnetic systems where spins accept more than two discrete values (they map onto a nonmagnetic multi-component mixture). The present SMSA can be applied to a soft XY and Heisenberg fluids (replacing the discrete external field constraint (Eqs. (9) and (18)) by its continuous spin counterpart, such as the Lovett equation [50], for instance). These questions as well as the problem of improving the thermodynamic self-consistency of the integral equation approach is left for future considerations.

\section{ACKNOWLEDGMENT}

Part of this work was supported by the Fonds zur Förderung der wissenschaftlichen Forschung under Project No. P15247. I.O. and I.M. thank the Fundamental Researches State Fund of the Ministry of Education and Science of Ukraine for support under Project No. $02.07 / 00303$. 


\section{REFERENCES}

[1] N. E. Frankel and C. J. Thompson, J. Phys. C: Solid State Phys. 8, 3194 (1975).

[2] P. C. Hemmer and D. Imbro, Phys. Rev. A 16, 380 (1977).

[3] J. M. Tavares, M. M. Telo da Gama, P. I. C. Teixeira, J. J. Weis, and M. J. P. Nijmeijer, Phys. Rev. E 52, 1915 (1995).

[4] F. Schinagl, H. Iro, and R. Folk, Eur. Phys. J. B 8, 113 (1999).

[5] F. Schinagl, R. Folk, and H. Iro, Condens. Matter Phys. 2, 313 (1999).

[6] W. Fenz and R. Folk, Phys. Rev. E 67, 021507 (2003).

[7] W. Fenz, R. Folk, I. M. Mryglod, and I. P. Omelyan, Phys. Rev. E 68, 061510 (2003).

[8] E. Lomba, J.J. Weis, N.G. Almarza, F. Bresme, and G. Stell, Phys. Rev. E 49, 5169 (1994).

[9] T. G. Sokolovska, Physica A 253, 459 (1998).

[10] F. Lado and E. Lomba, Phys. Rev. Lett. 80, 3535 (1998).

[11] F. Lado, E. Lomba, and J. J. Weis, Phys. Rev. E 58, 3478 (1998).

[12] T. G. Sokolovska and R. O. Sokolovskii, Phys. Rev. E 59, R3819 (1999).

[13] E. Lomba, F. Lado, and J. J. Weis, Condens. Matter Phys. 4, 45 (2001).

[14] D. Marx, P. Nielaba, and K. Binder, Phys. Rev. Lett. 67, 3124 (1991).

[15] M. J. P. Nijmeijer and J. J. Weis, in Annual Review of Computational Physics IV, edited by D. Stauffer (World Scientific, Singapore, 1996).

[16] M. J. P. Nijmeijer and J. J. Weis, Phys. Rev. E 53, 591 (1996).

[17] J. J. Weis, M. J. P. Nijmeijer, J. M. Tavares, and M. M. Telo da Gama, Phys. Rev. E 55, 436 (1997).

[18] M. J. P. Nijmeijer, A. Parola, and L. Reatto, Phys. Rev. E 57, 465 (1998).

[19] A. L. Ferreira and W. Korneta, Phys. Rev. E 57, 3107 (1998).

[20] I. M. Mryglod, I. P. Omelyan, and R. Folk, Phys. Rev. Lett. 86, 3156 (2001).

[21] T. Kawasaki, Prog. Theor. Phys. 58, 1357 (1977).

[22] R. O. Sokolovskii, Phys. Rev. B 61, 36 (2000).

[23] S. Romano and R. O. Sokolovskii, Phys. Rev. B 61, 11379 (2000).

[24] N. B. Wilding, F. Schmid, and P. Nielaba, Phys. Rev. E 58, 2201 (1998). 
[25] G. Kahl, E. Schöll-Paschinger, and A. Lang, Monatshefte für Chemie 132, 1413 (2001).

[26] E. Schöll-Paschinger, D. Levesque, J. J. Weis, and G. Kahl, Phys. Rev. E 64, 011502 (2001).

[27] O. Antonevych, F. Forstmann, and E. Diaz-Herrera, Phys. Rev. E 65, 061504 (2002).

[28] G. Kahl, E. Schöll-Paschinger, and G. Stell, J. Phys.: Condens. Matter 14, 9153 (2002).

[29] E. Schöll-Paschinger and G. Kahl, J. Chem. Phys. 118, 7414 (2003).

[30] D. Pini, M. Tau, A. Parola, and L. Reatto, Phys. Rev. E 67, 046116 (2003).

[31] N. B. Wilding, Phys. Rev. E 67, 052503 (2003).

[32] J.P. Hansen and I.R. McDonald, Theory of Simple Liquids, 2nd edn. (Academic: London, 1986).

[33] D. Pini, G. Stell, N. B. Wilding, Mol. Phys. 95, 483 (1998).

[34] A. Parola and L. Reatto, Adv. Phys. 44, 211 (1995).

[35] M. Tau, A. Parola, D. Pini, and L. Reatto, Phys. Rev. E 52, 2644 (1995).

[36] A. Brognara, Thesis of Universita degli Studi di Milano, 2001/2002.

[37] L. Blum and J. S. Høye, J. Stat. Phys. 19, 317 (1978).

[38] E. Arrieta, C. Jedrzejek, and K. N. Marsh, J. Chem. Phys. 95, 6806 (1991).

[39] Y. Tang, J. Chem. Phys. 118, 4140 (2003).

[40] J.L. Lebowitz and J.K. Percus, Phys. Rev. 144, 251 (1966).

[41] W. G. Madden and S. A. Rice, J. Chem. Phys. 72, 4208 (1980).

[42] N. Choudhury and S. K. Ghosh, J. Chem. Phys. 116, 8517 (2002).

[43] A. Kovalenko and F. Hirata, Chem. Phys. Lett. 349, 496 (2001).

[44] A. Kovalenko and F. Hirata, J. Chem. Phys. 110, 10095 (1999).

[45] J.G. Kirkwood, J. Chem. Phys. 3, 300 (1935).

[46] A. Kovalenko, S. Ten-no, and F. Hirata, J. Comput. Chem. 20, 928 (1999).

[47] B. Groh and S. Dietrich, Phys. Rev. Lett. 72, 2422 (1994).

[48] P. H. E. Meijer, M. Keskin and I. L. Pegg, J. Chem. Phys. 88, 1976 (1988).

[49] P. H. von Konynenburg and R. L. Scott, Philos. Trans. R. Soc. London A298, 495 (1980).

[50] R. Lovett, C. Y. Mou, and F. P. Buff, J. Chem. Phys. 65, 570 (1976). 


\section{FIGURE CAPTIONS}

FIG. 1. The liquid-gas coexistence densities $\rho^{*}$ as a function of temperature $T^{*}$ obtained within the OZ/SMSA/FC integral equation approach (bold curves) for the soft-core ideal $(R=\infty)$ Ising fluid with $z_{1}^{*}=1$ and different values of the external field, $H^{*}=0,0.1,0.5$,

1,5 , and $\infty$, (subsets (a), (b), (c), (d), (e), and (f), respectively). The results of the usual and the adjustable versions [7] of the SCMF theory are plotted correspondingly by thin and dashed curves. The Gibbs ensemble MC simulation data [7] are shown as circles. The para-ferro magnetic phase transition (at $H^{*}=0$, subset (a)) is shown by the long dashed curve.

FIG. 2. The liquid-gas coexistence densities $\rho^{*}$ as a function of temperature $T^{*}$ obtained within the OZ/SMSA/FC integral equation approach for the soft-core ideal Ising fluid at different values, $z_{1}^{*}=0.5,1,2$, and 3 , of the inverse screening length (subsets (a), (b), (c), and (d), respectively) as well as different values of the external field, namely, top to bottom and alternating solid and dashed curves, $H^{*}=0,0.01,0.1,0.3,0.5,1,2$, and 5 (within each subset). At $z_{1}^{*}=3$, an additional curve corresponding to $H^{*}=9$ is included in subset (d). Note that the curves corresponding to $H^{*}=\infty$ are not shown, because they practically coincide with those related to $H^{*}=5$ at $z_{1}^{*}=0.5,1$, and 2 (subsets (a), (b), and (c)) or to $H^{*}=9$ at $z_{1}^{*}=3$ (subsets $(\mathrm{d})$ ). The para-ferro magnetic phase transition (at $H^{*}=0$ ) is plotted in the subsets by the long dashed line.

FIG. 3. The critical temperature $T_{\mathrm{c}}^{*}(\operatorname{subset}(\mathrm{a}))$ and critical density $\rho_{\mathrm{c}}^{*}(\operatorname{subset}(\mathrm{b})$ ) as functions of the external field $H^{*}$, evaluated within the OZ/SMSA/FC approach for the ideal Ising fluid at various values of the inverse screening length $z^{*}$. At $z^{*} \rightarrow 0$, the result corresponds to the SCMF theory.

FIG. 4. (a) The para-ferro magnetic transition temperature $T_{\lambda}^{*}$ obtained at $H^{*}=0$ within the OZ/SMSA/FC integral equation approach for the soft-core ideal Ising fluid for different values of inverse screening length, from right to left, $z^{*}=0.5,1,2,3$, and 5 . The 
result of the standard HSMF theory is plotted as the dashed straight line and relates to the case $z^{*} \rightarrow 0$. (b) The results of the SCMF and OZ/SMSA/FC theories for the case $z^{*}=1$ are shown as dashed and solid curves, respectively, in comparison with canonical MC simulation data (circles) taken from Ref. [7].

FIG. 5. The gas-liquid coexistence densities $\rho^{*}$ as a function of temperature $T^{*}$ obtained within the OZ/SMSA/FC approach for the soft-core nonideal Ising fluid with $z_{1}^{*}=z_{2}^{*}=1$ at $H^{*}=0$ for larger values of $R$.

FIG. 6. The coexistence densities $\rho^{*}$ as a function of temperature $T^{*}$ obtained within the OZ/SMSA/FC approach for the soft-core nonideal Ising fluid with $z_{1}^{*}=z_{2}^{*}=1$ at $H=0$ for moderate (subset (a)) and small (subset (b)) values of $R$.

FIG. 7. The coexistence densities $\rho^{*}$ as a function of temperature $T^{*}$ obtained within the OZ/SMSA/FC approach for the nonideal Ising fluid at $R=0.215$ (subset (a)) and $R=0.14$ (subset (b)) corresponding to different values, $z_{1}^{*}=z_{2}^{*}=0.5,1,2$, and 3 , of the inverse screening radii.

FIG. 8. The same as in Fig. 7 but for $z_{1} \neq z_{2}$ at $R=0.215$ (subsets (a) and (b)) and $R=0.14$ (subsets $(\mathrm{c})$ and $(\mathrm{d}))$.

FIG. 9. The complete thermodynamic phase diagrams of the nonideal Ising fluid with $z_{1}^{*}=z_{2}^{*}=1$ evaluated using the OZ/SMSA/FC approach and projected onto the $\left(T^{*}, \rho^{*}\right)$ plane at some typical values of $R=5,1,0.29,0.215,0.2,0.19,0.16$, and 0.12 (subsets (a), (b), (c), (d), (e), (f), (g), and (h), respectively). The families of the diagrams in each of the subsets correspond to different values of the external field, $H^{*}=0,0.01,0.1,0.5,1,2,3,5$, 9 , and $\infty$ (the value $H^{*}=0.3$ for $R=5$ and 1 , as well as $H^{*}=20$ for $R=0.12$ are included additionally, whereas $H^{*}=9$ is excluded for $R=5$ and 1 ).

FIG. 10. The critical temperature of the wing lines $T_{\mathrm{c}}^{*(w)}$, subsets (a) and (b), and the gas-liquid critical lines $T_{\mathrm{c}}^{*(g l)}$, subsets (c) and (d), as a function of the external magnetic field $H^{*}$ for the nonideal Ising fluid at different values of parameter $R$. 
FIG. 11. The critical density of the wing lines $\rho_{\mathrm{c}}^{*(w)}$, subsets (a) and (b), and the gasliquid critical lines $\rho_{\mathrm{c}}^{*(g l)}$, subsets $(\mathrm{c})$ and $(\mathrm{d})$, as a function of the external magnetic field $H^{*}$ for the nonideal Ising fluid at different values of $R$.

FIG. 12. The thermodynamic phase diagrams obtained within the OZ/SMSA/FC approach for the soft-core nonideal Ising fluid with $z_{1}^{*}=z_{2}^{*}=1$ at $R=R_{v l}=0.196$. The phase coexistence curves projected onto the $\left(T^{*}, \rho^{*}\right)$ plane are shown in subset (a) for different external field values, $H^{*}=0,0.01,0.1,0.5,1,2,3,5,9$, and $\infty$. A more detailed phase behavior near the asymmetric tricritical point is presented in subset (b) for (bottom to top) $H^{*}=1,1.2,1.4,1.6,1.8,2,2.2$, and 2.6. Here, the gas-liquid (on the left) and wing line (on the right) critical points (circles) are connected by thin curves. The critical temperature $T_{\mathrm{c}}^{*(g l, w)}$ and critical density $\rho_{\mathrm{c}}^{*(g l, w)}$ of the gas-liquid and liquid-liquid phase transitions are plotted as functions of $H^{*}$ in subset (c) and (d), respectively. They meet in the tricritical point (dots).

FIG. 13. Global phase diagram of an Ising fluid. Type I contains at $H=0$ a tricritical point, where the magnetic transition line and two wing lines, existing for arbitrary $\pm H$, meet. Type IIa contains a tricritical point (at $H=0$ ) and the gas-liquid critical lines (for $\pm H$ ), ending in a critical end point at finite magnetic field, while the wing lines exists for arbitrary magnetic field. Type IIb contains a tricritical point (at $H=0$ ) and the gas-liquid critical lines (for $\pm H$ ) extending to infinite magnetic field, whereas the wing lines end in a critical end point at finite magnetic field. Type III contains only gas-liquid critical lines extending to infinite values of magnetic field, and the magnetic transition line ends in a critical end point at $H=0$.

FIG. 14. The para-ferro magnetic coexistence curves evaluated at $H=0$ using the $\mathrm{OZ} / \mathrm{SMSA} / \mathrm{FC}$ theory for the soft-core nonideal Ising fluid with $z_{1}^{*}=z_{2}^{*}=1$ at different values of the system parameter, namely, top to bottom, $R=0.1,0.12,0.14,0.17,0.215$, $0.29,0.4$, and $\infty$. The results of the HSMF and SCMF approaches are plotted as the shortand long-dashed curves, respectively. 

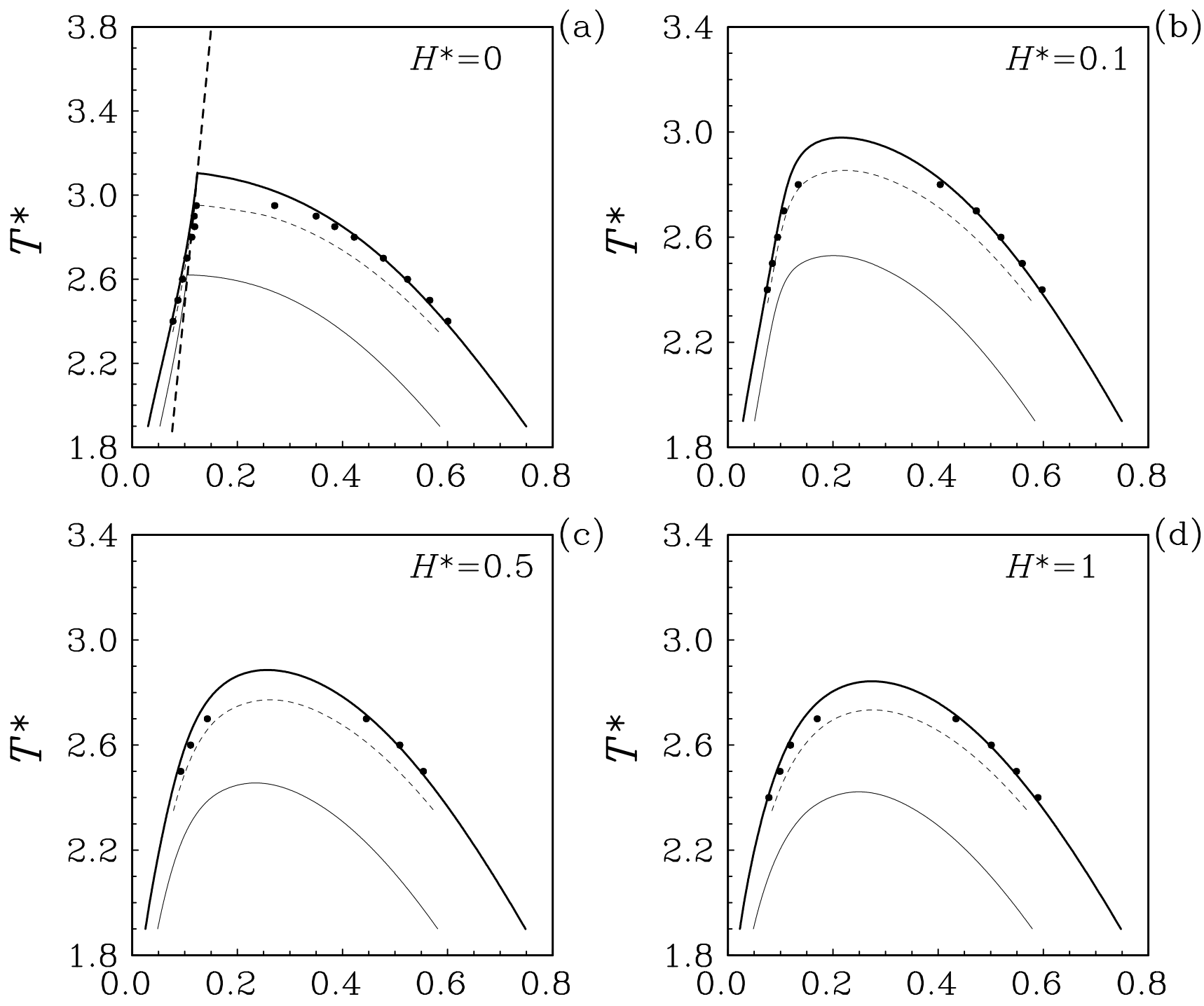

(c)
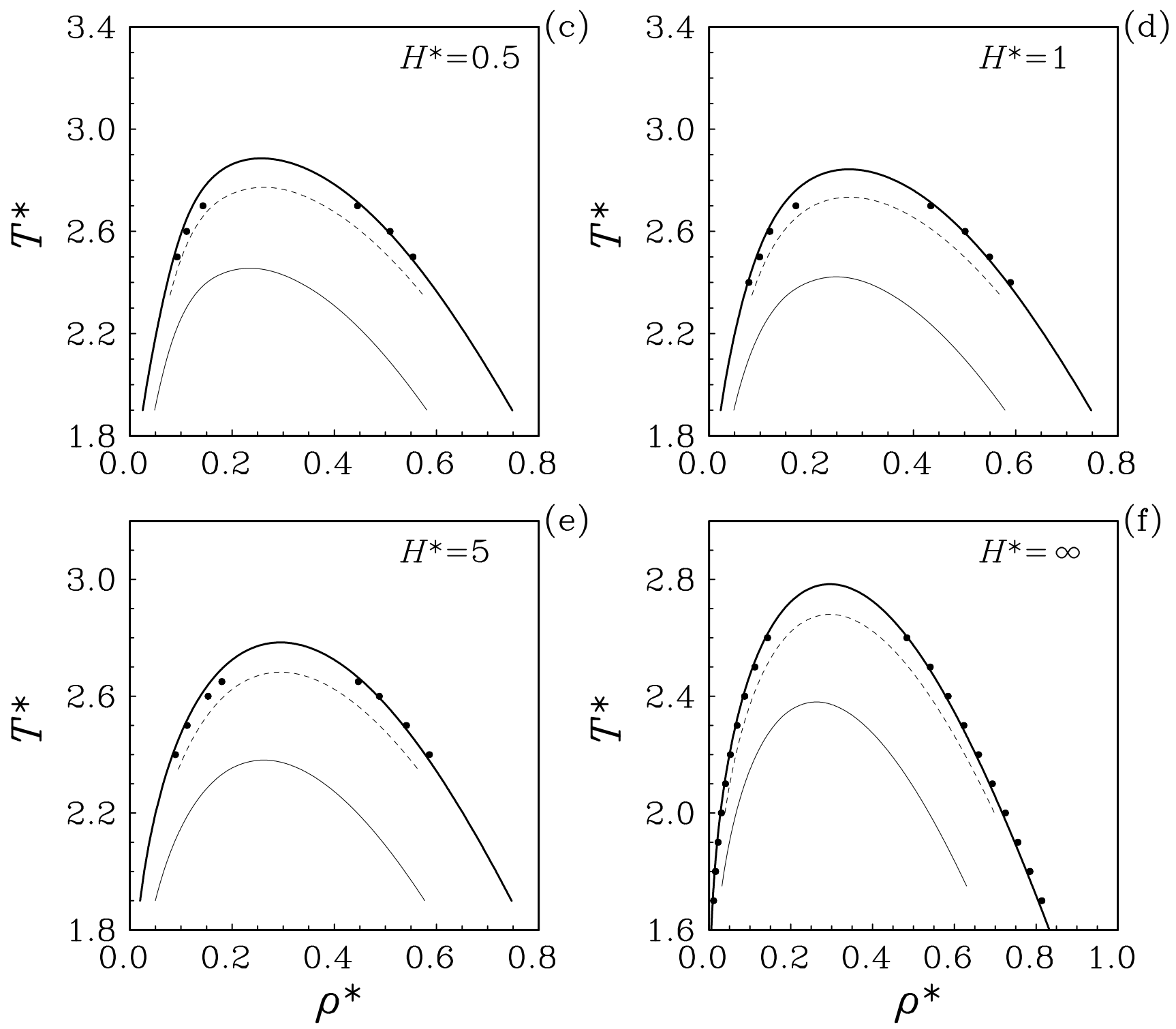


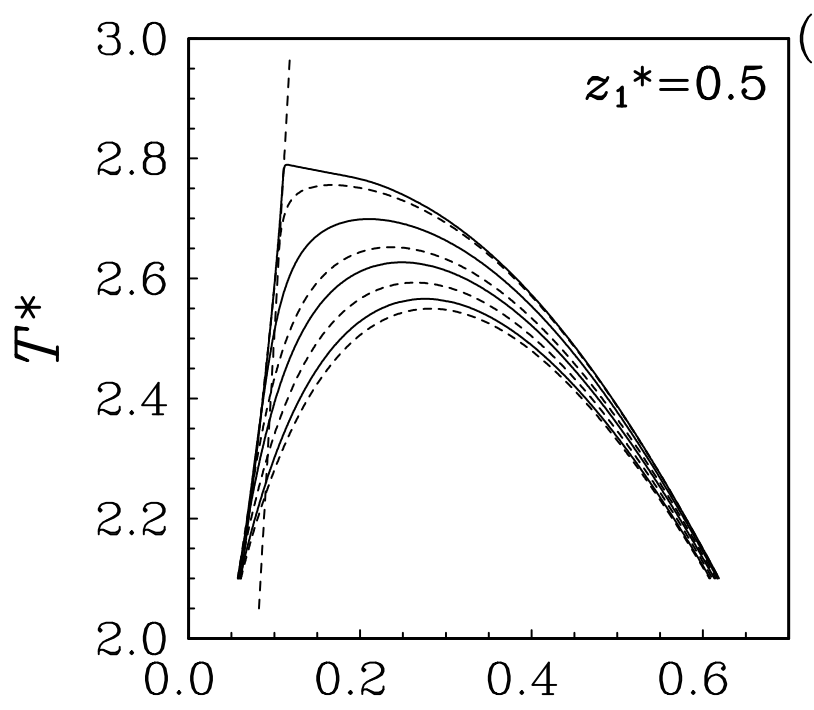

(a)
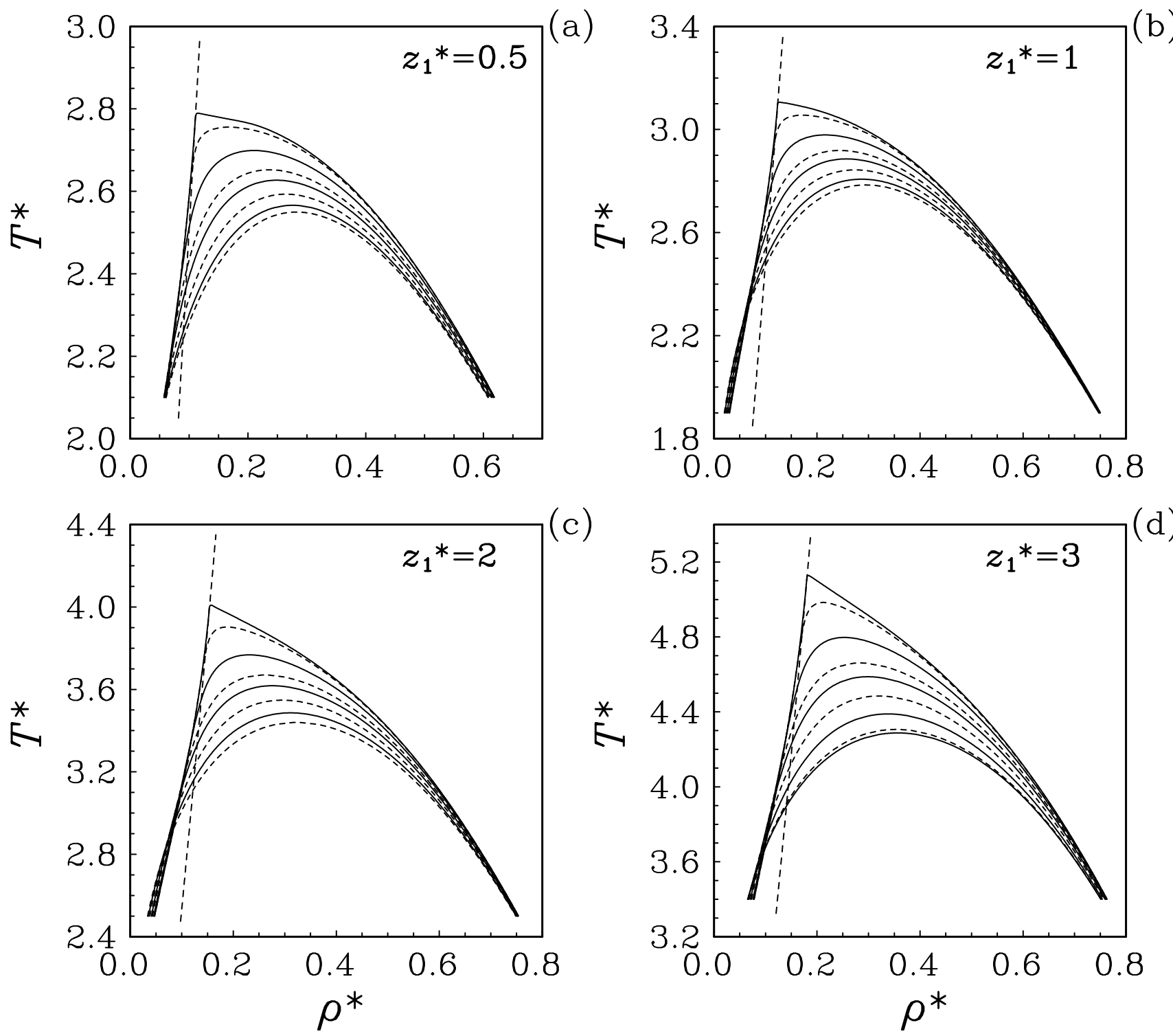

(c)

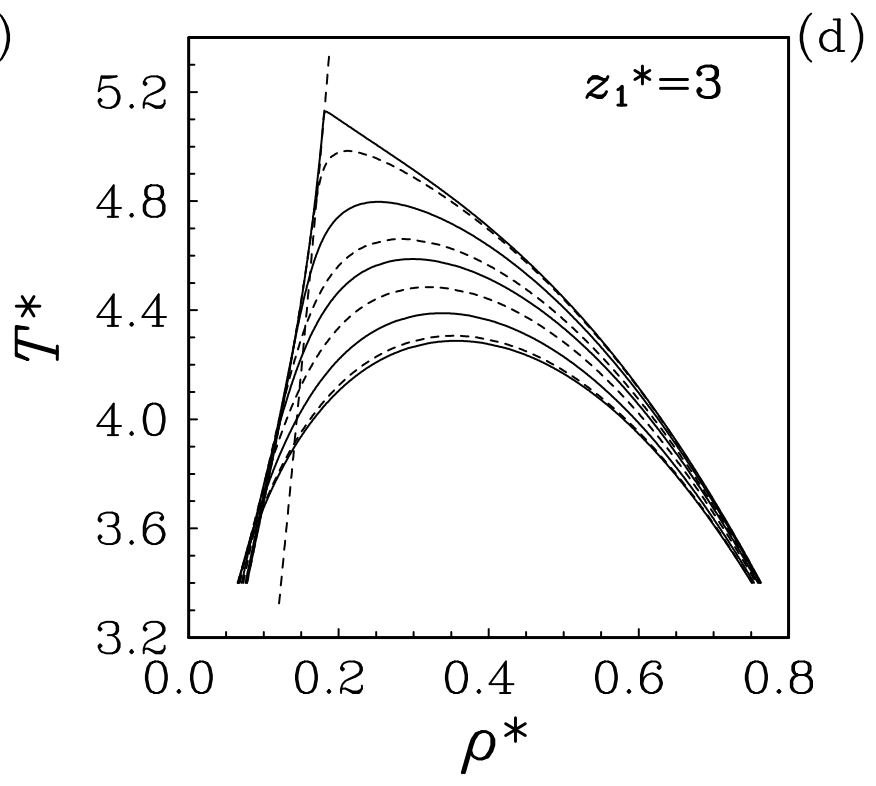



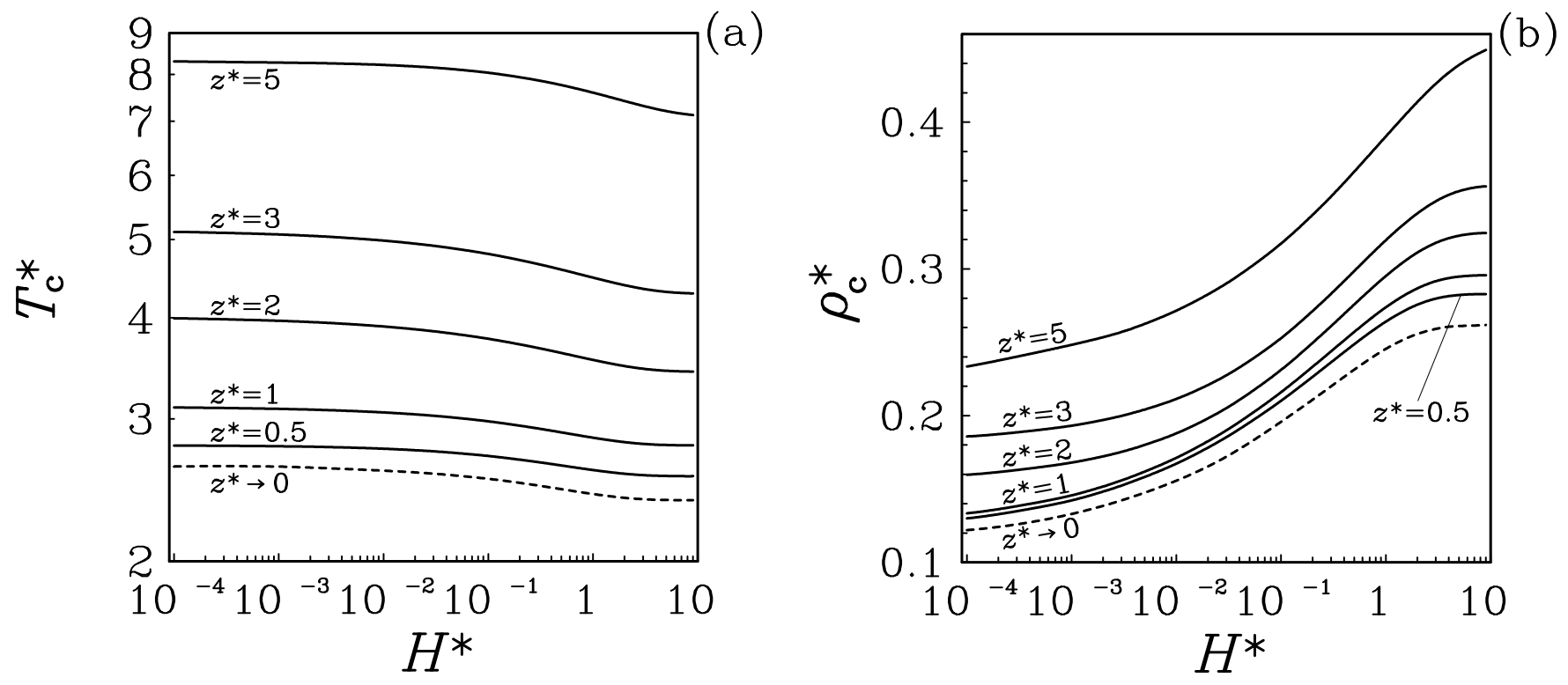

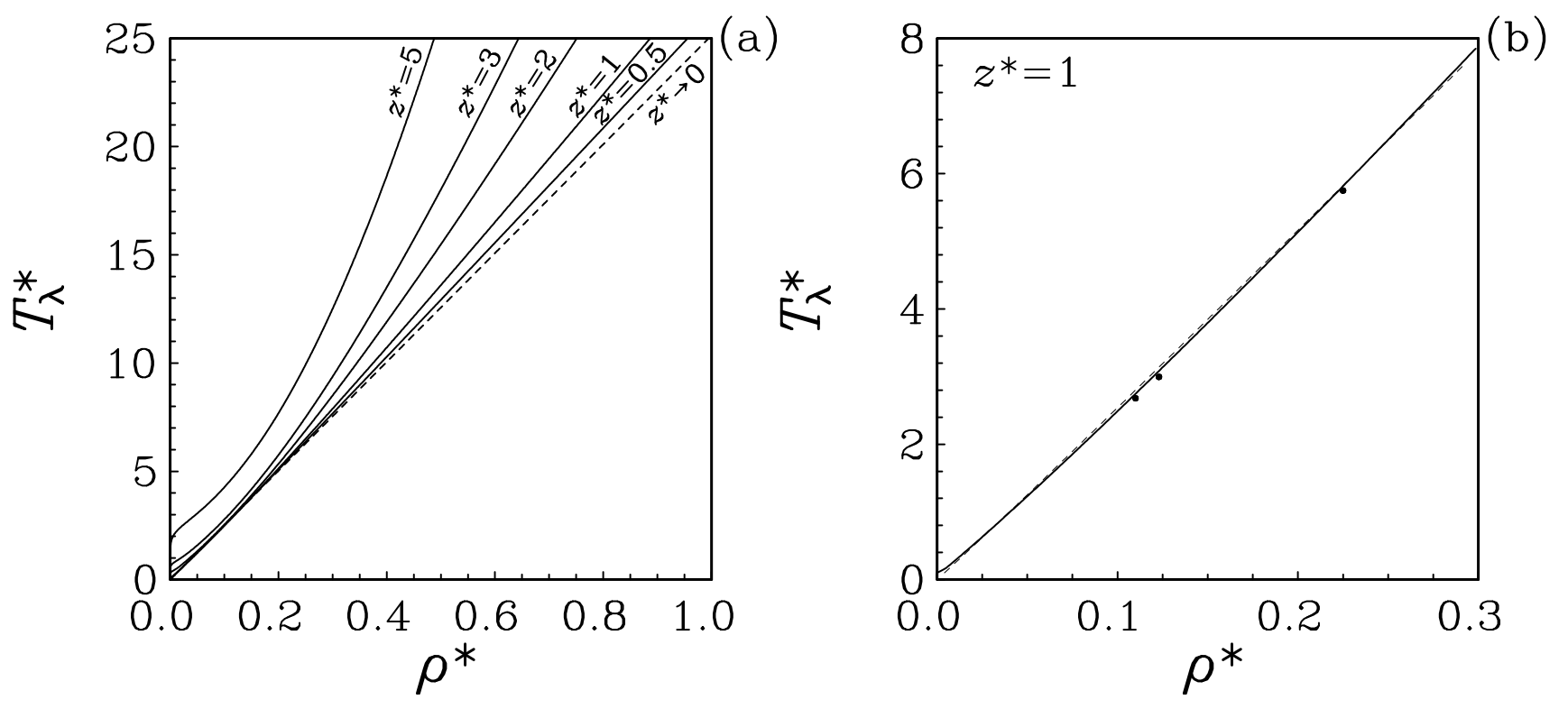


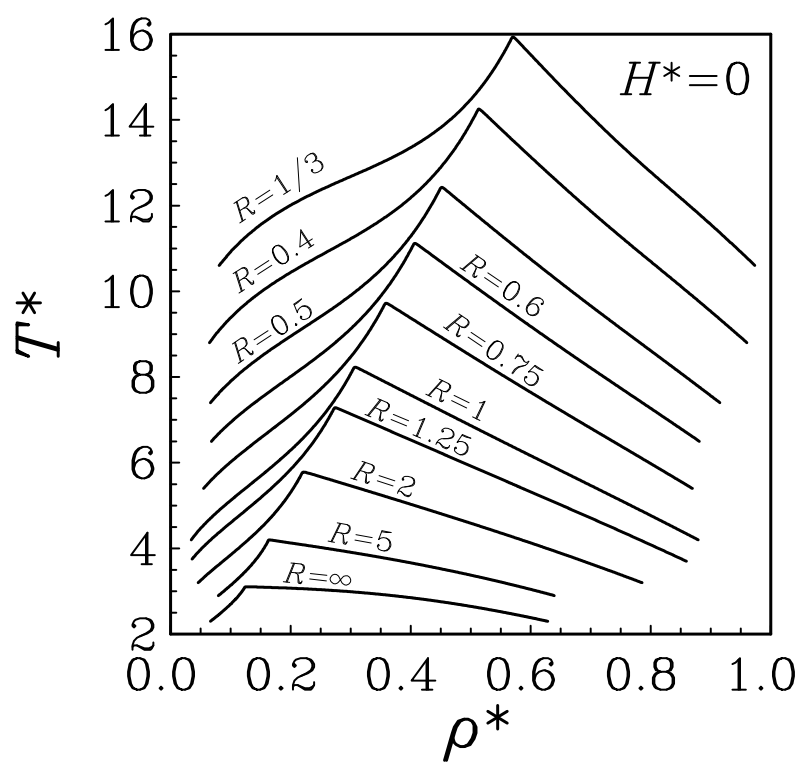



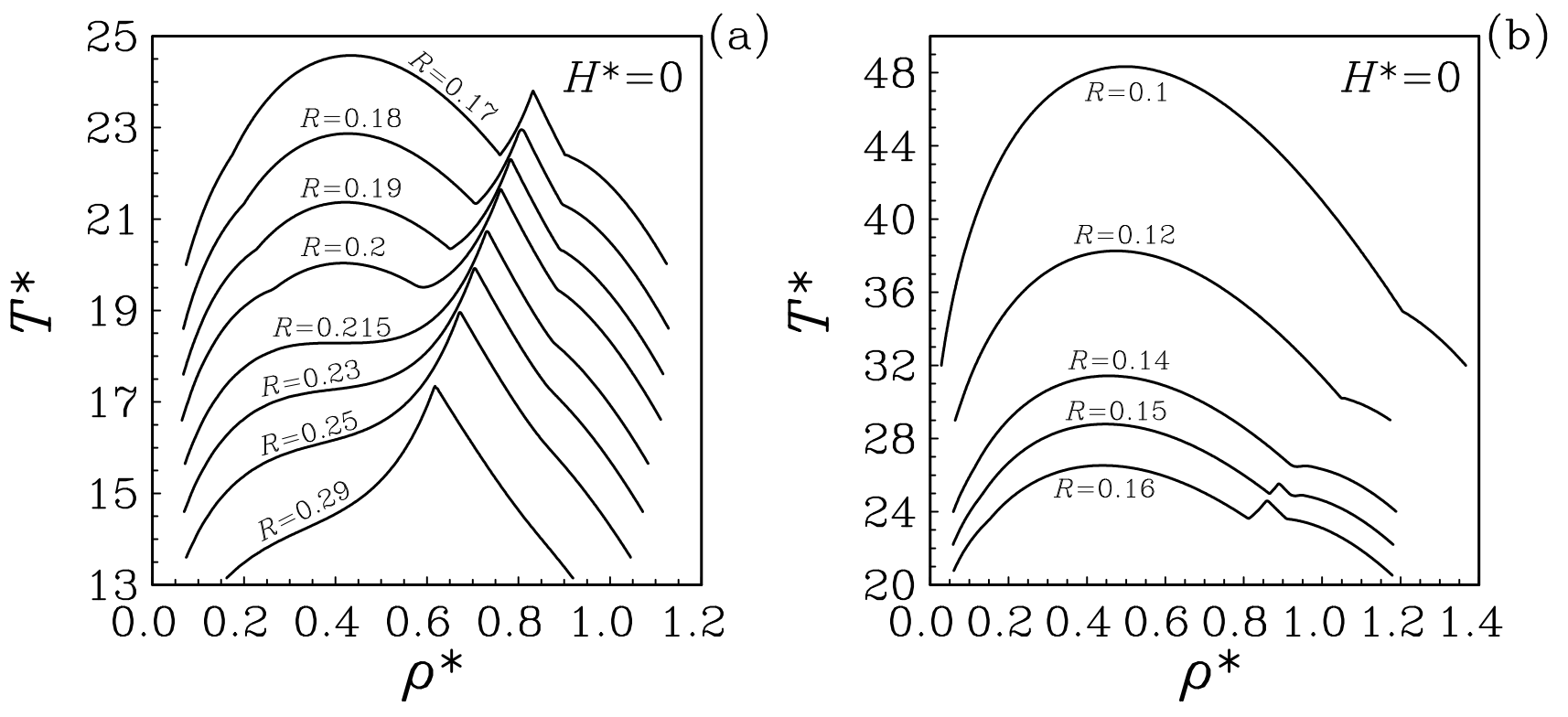

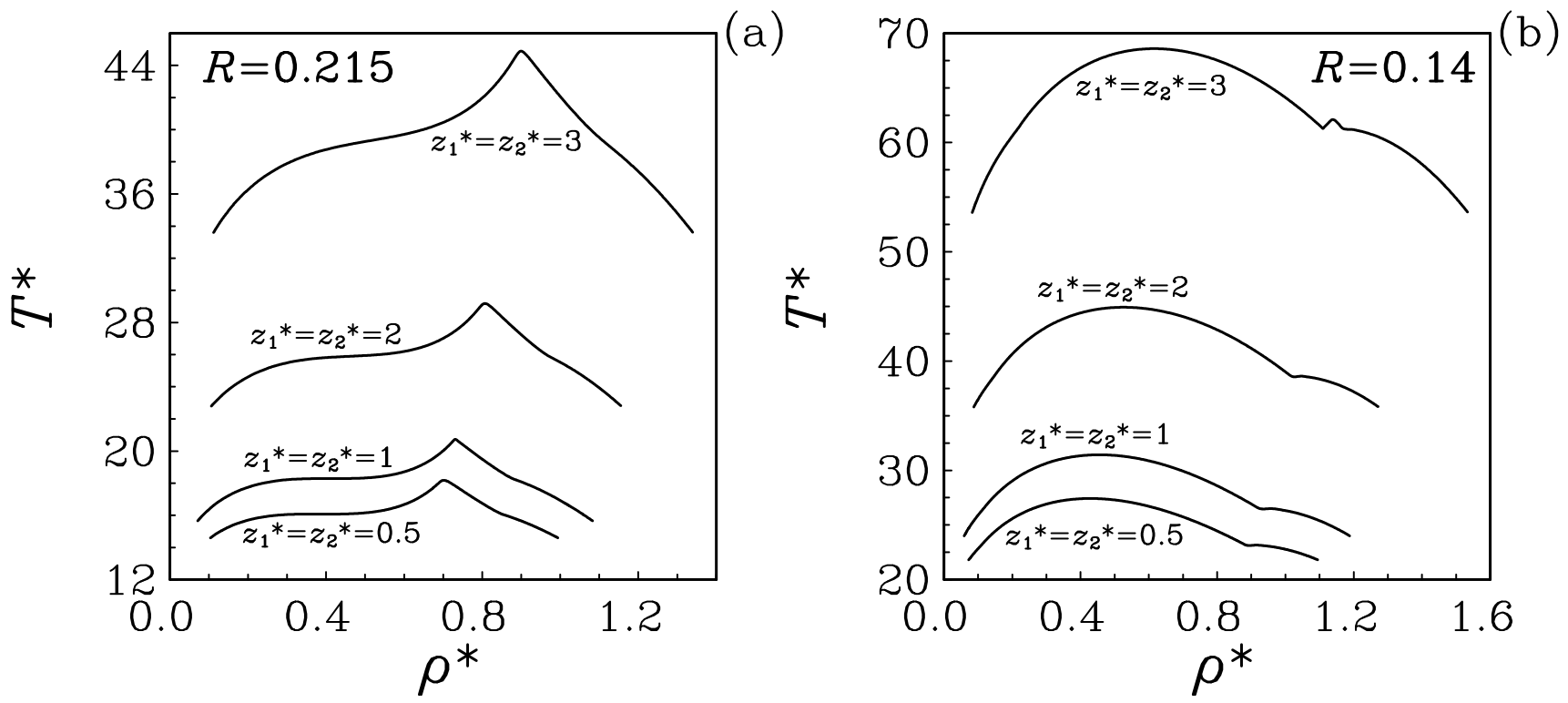


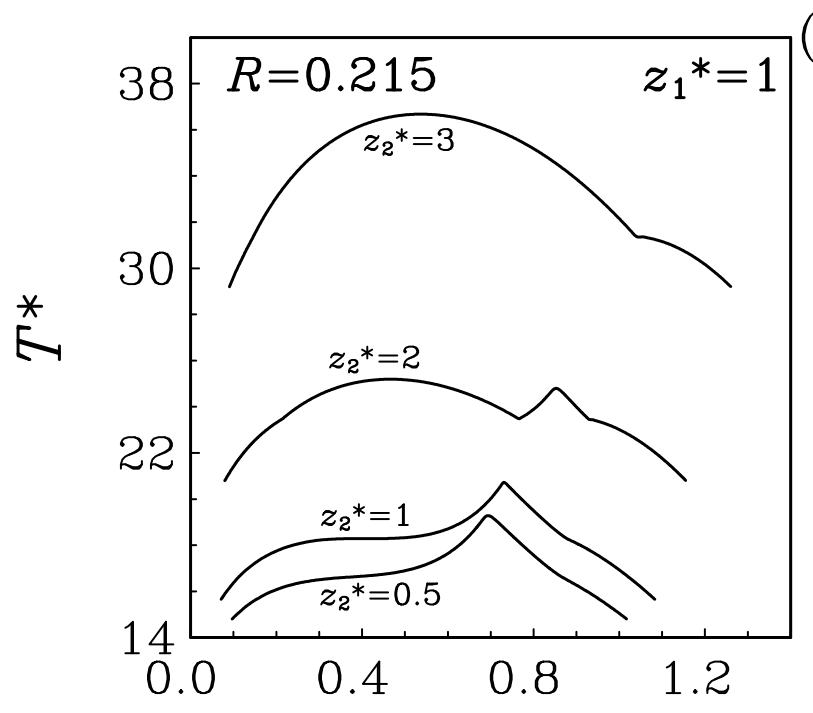

(a)
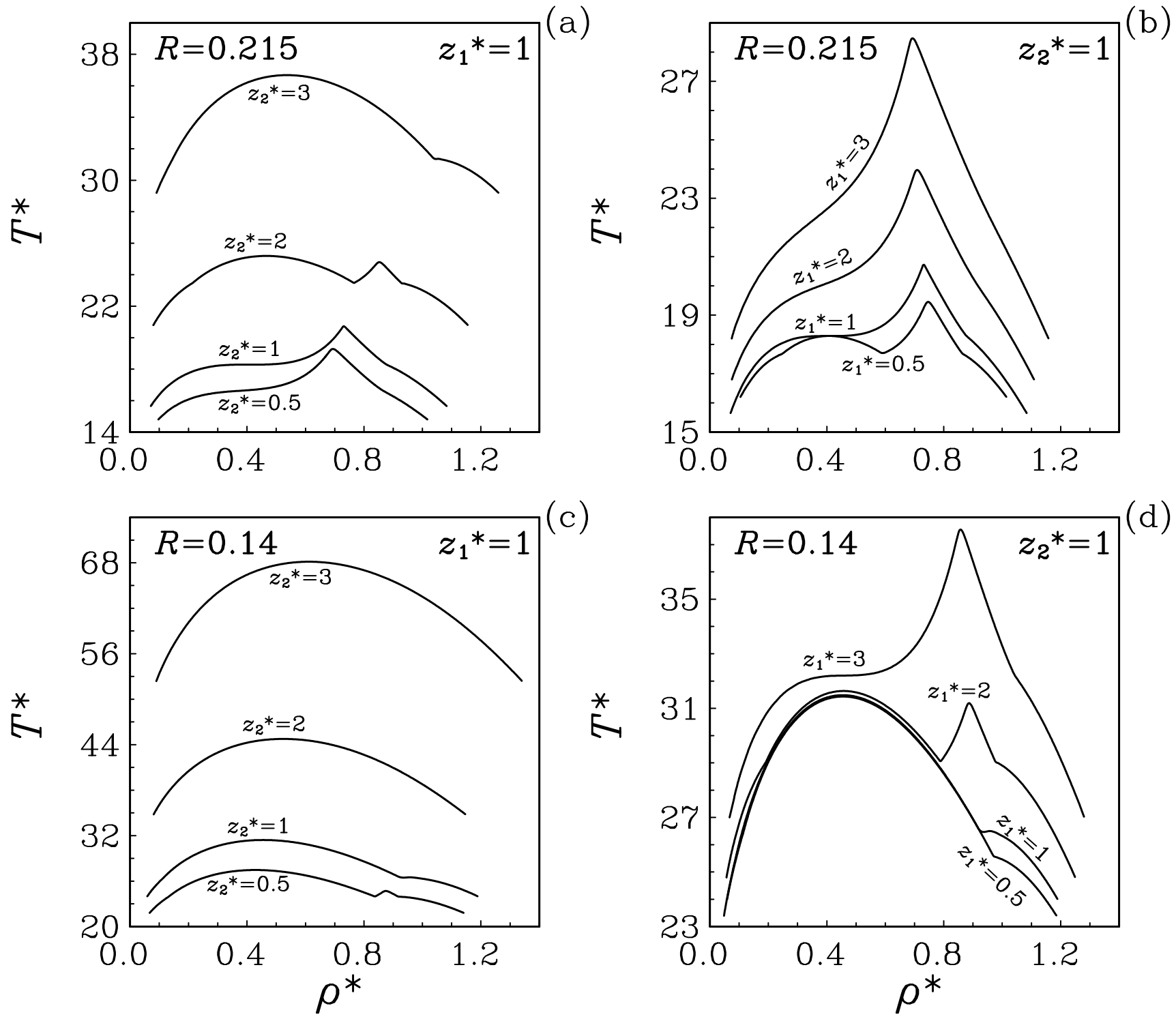

(c)

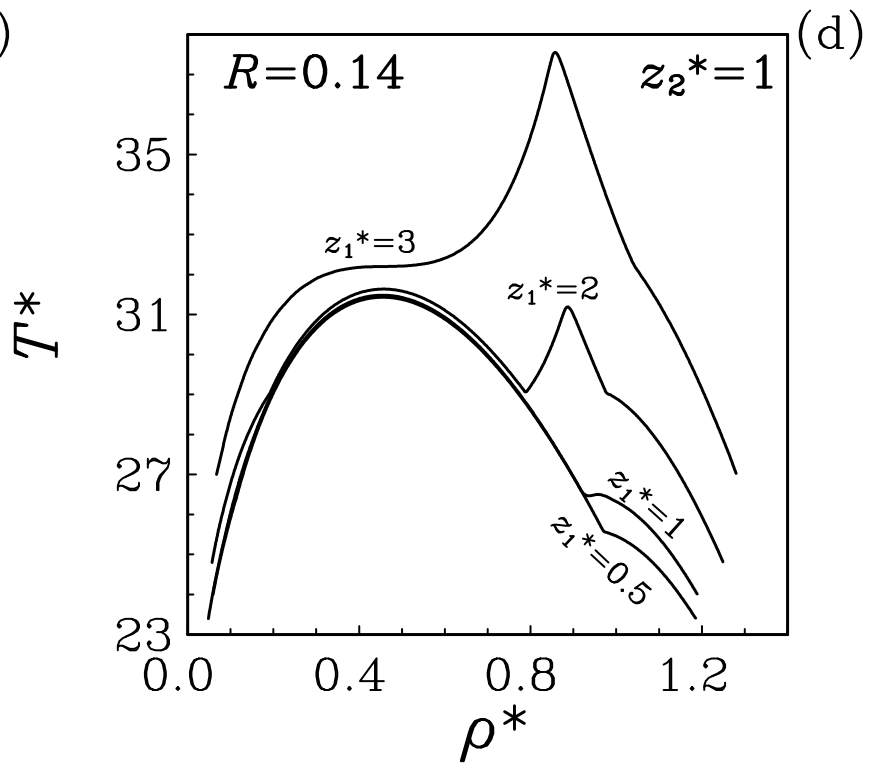



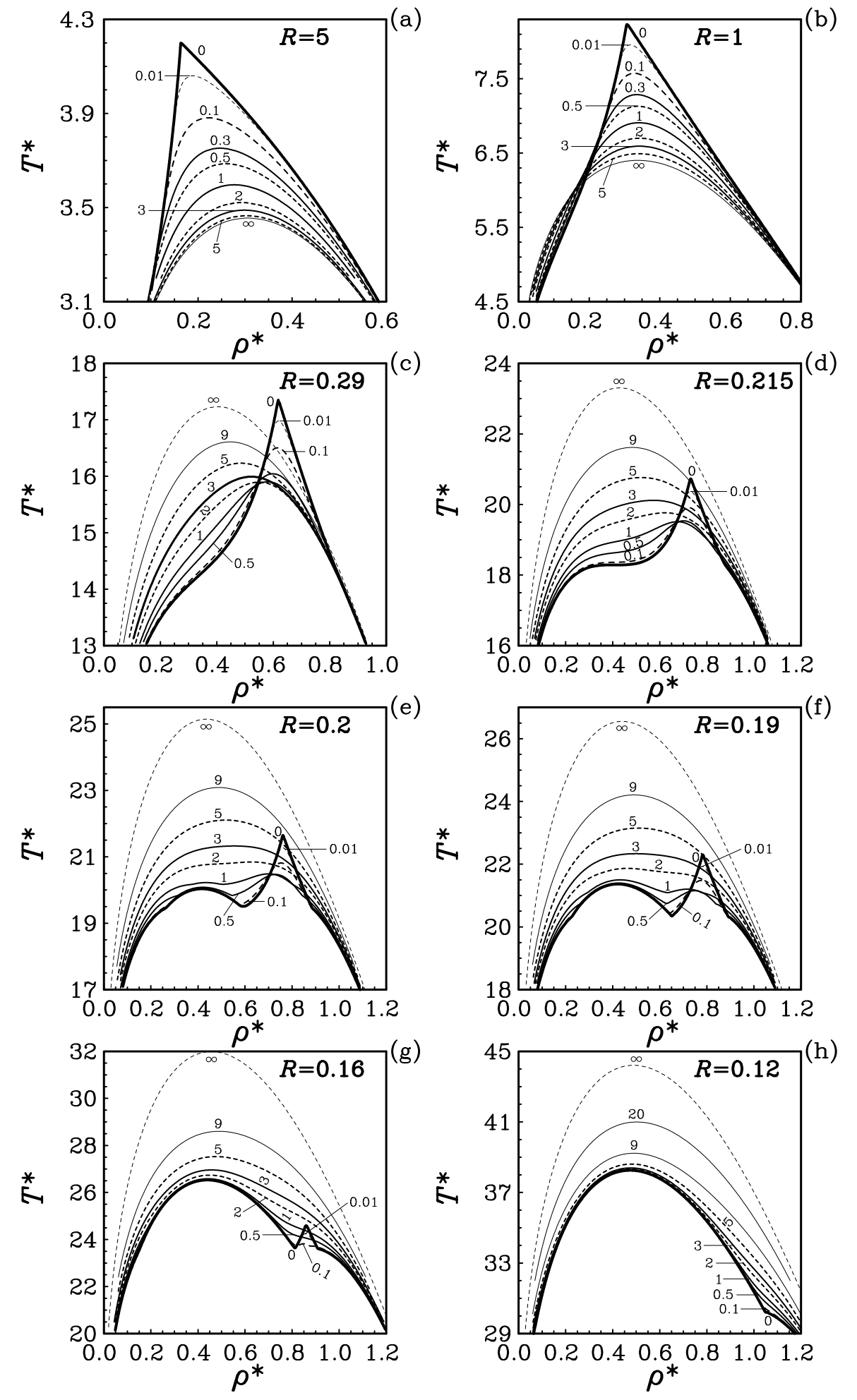

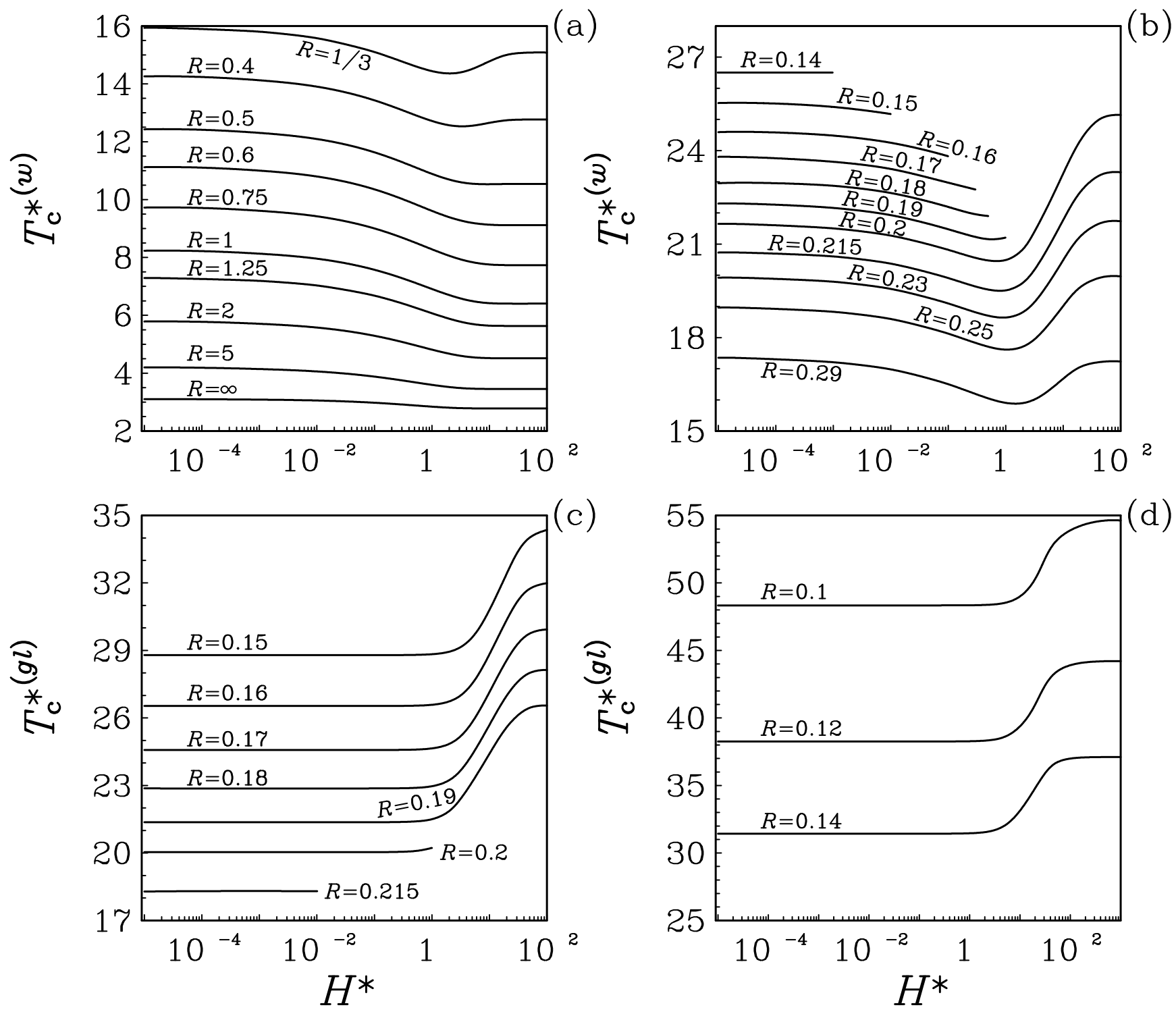

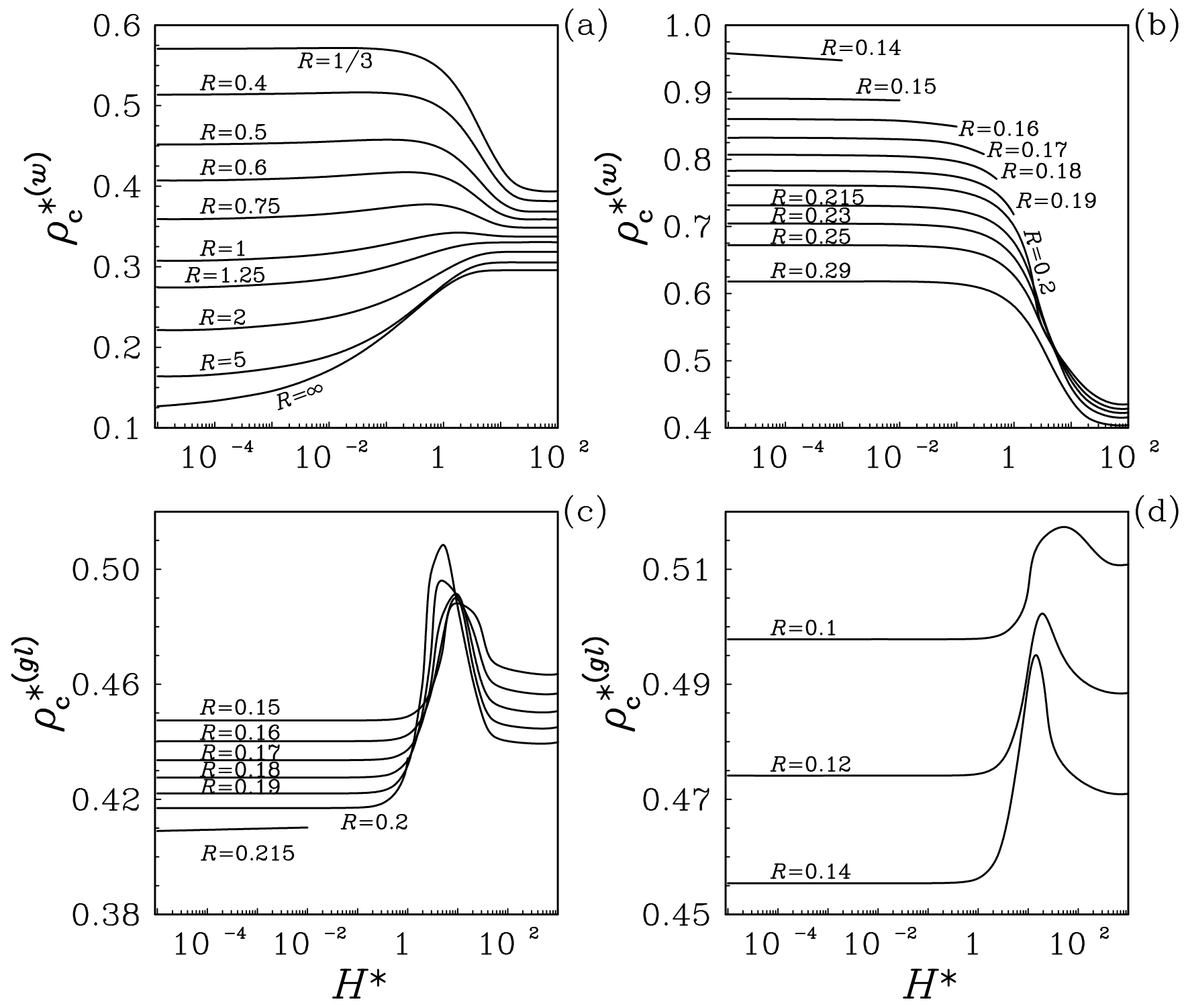

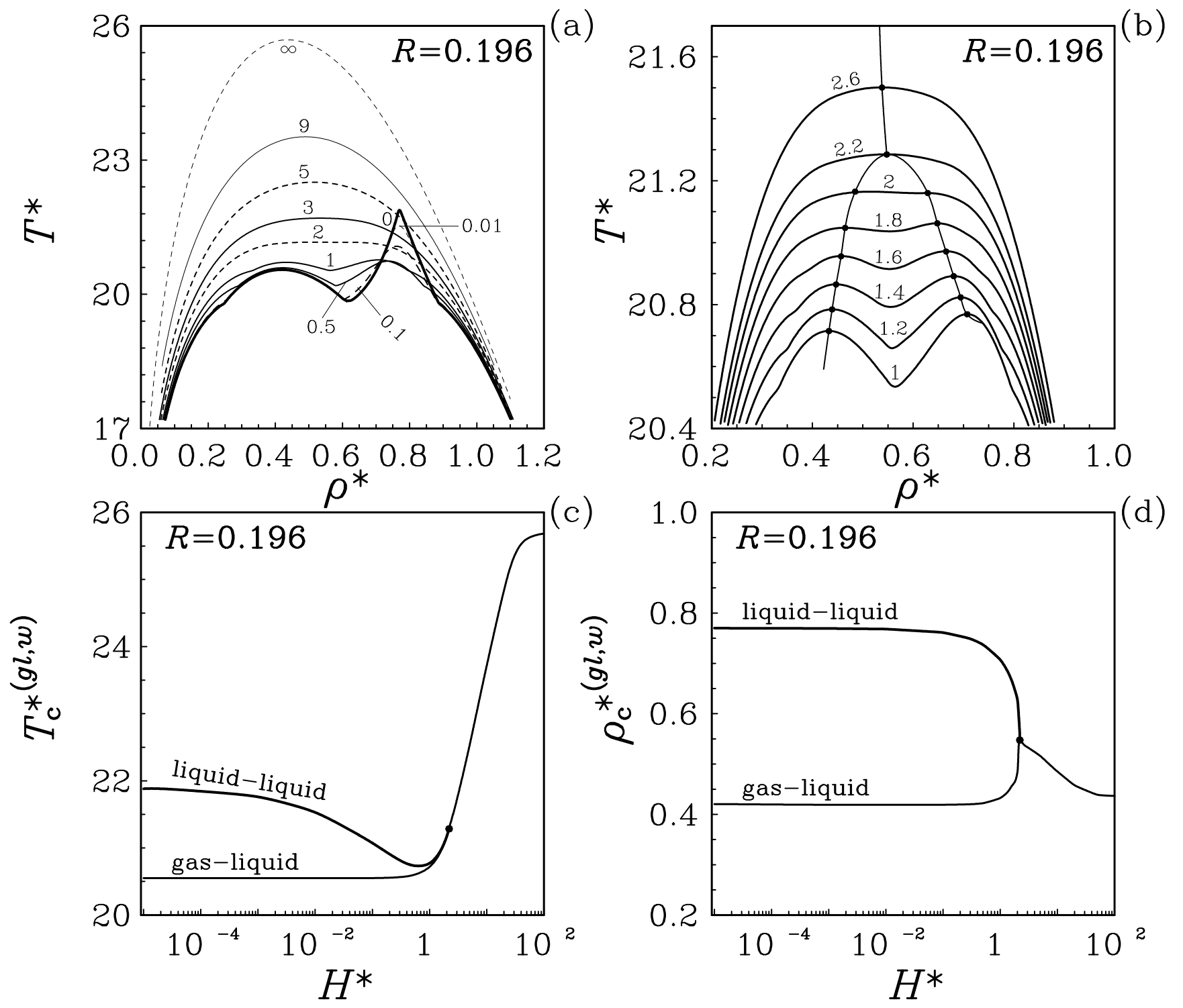

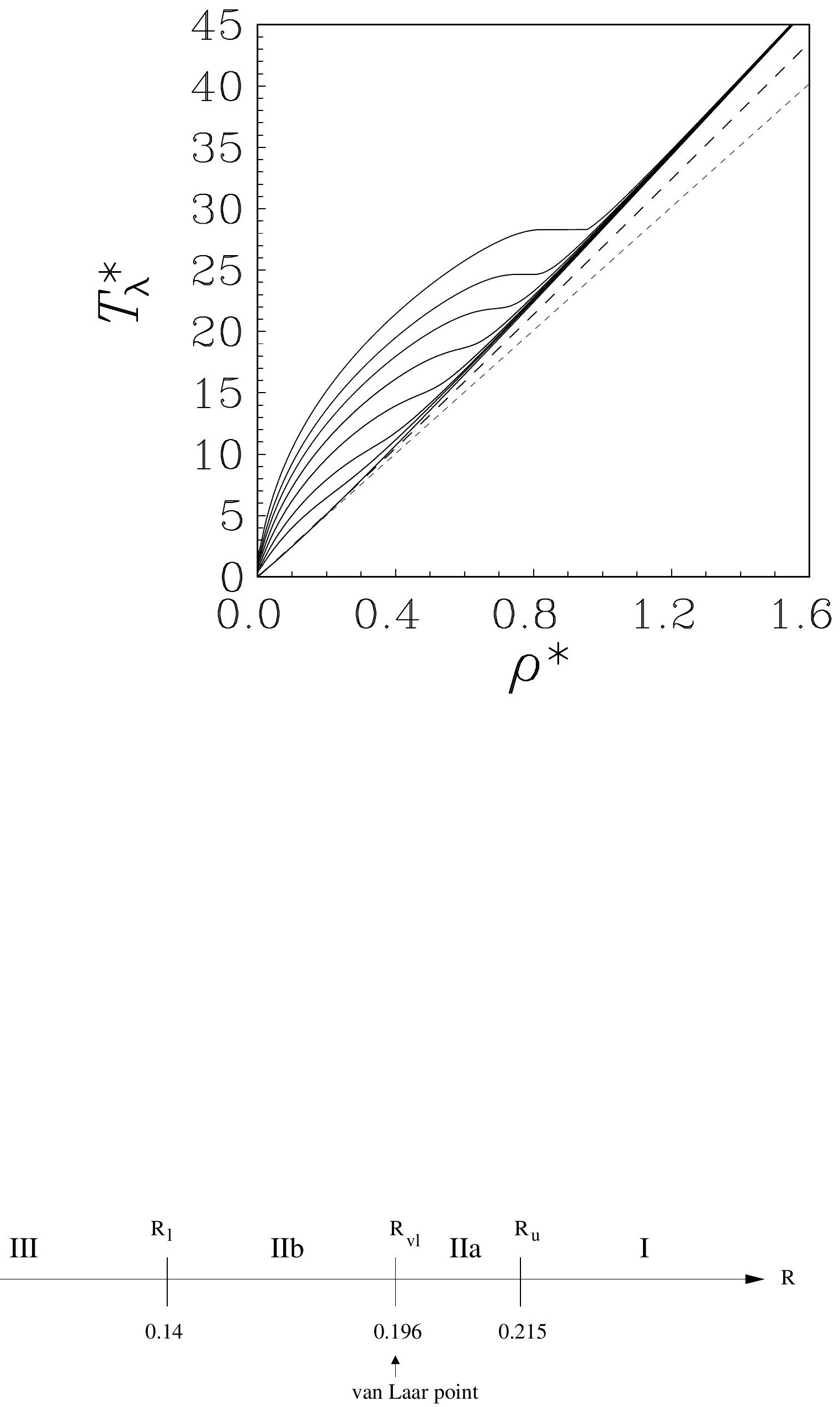